\title{
The Effects of Model Aromatic Lignin Compounds on Growth and Lipid Accumulation of Rhodococcus rhodochrous
}

Sara A. Shields-Menard (1), Marta AmirSadeghi (2), Magan Green (3), Erika Womack

(4), Darrell L. Sparks (5), Jacqui Blake (6), Mariola Edelmann (7), Xuan Ding (8), Badamkhand Sukhbaatar (9), Rafael Hernandez (10), Janet R. Donaldson (11), Todd French (6)*

(1) Department of Biological Sciences, Nicholls State University, Thibodaux, LA

(2) Chemical \& Materials Engineering, California State Polytechnic University, Pomona, CA

(3) Mississippi State Chemical Lab, Mississippi State University, Mississippi State, MS

(4) USDA-ARS, Mississippi State, MS

(5) Department of Biochemistry, Molecular Biology, Entomology, and Plant Pathology, Mississippi State University, Mississippi State, MS

(6) Dave C. Swalm School of Chemical Engineering, Mississippi State University, Mississippi State, MS

(7) Department of Microbiology and Cell Science, College of Agricultural and Life Sciences, University of Florida, Gainesville, FL

(8) Institute for Genomics, Biocomputing, and Biotechnology, Mississippi State University, Mississippi State, MS

(9) Mississippi Gulf Coast Community College, MS

(10) Department of Chemical Engineering, University of Louisiana at Lafayette, Lafayette, LA

(11) Department of Biological Sciences, The University of Southern Mississippi, Hattiesburg, MS

* Corresponding authors: French@che.msstate.edu

W. Todd French

french@che.msstate.edu

662-325-4308 (office)

662-325-2482 (fax) 


\begin{abstract}
Lignocellulosic biomass is one of the most abundant and renewable organic materials in the world. The lignocellulosic complex is composed of cellulose, hemicellulose, and lignin, which can be pretreated to release sugars that can be utilized for microbial production of valued metabolites. Oleaginous microbes can accumulate over $20 \%$ of their cell dry weight as lipids, which are stored as intracellular energy reserves. The characterization of oleaginous bacteria creates opportunities for the development of alternative feedstocks and technologies. Rhodococcus rhodochrous is a bacterium recently determined to be oleaginous when grown in glucose-supplemented media. The purpose of this study was to evaluate model lignin phenolic compounds as substrates for lipid accumulation. Lipid accumulation in $R$. rhodochrous was evaluated using phenol, 4hydroxybenzoic acid (HBA) and vanillic acid (VA) as model lignin compounds with and without glucose as a co-substrate. Cell dry weight increased in all treatments, indicating that growth was not impaired in these conditions. However, alterations were observed in the amount of lipids produced. Dry cell weight and lipids were analyzed daily. $R$. rhodochrous accumulated over $40 \%$ of its cell dry weight as lipids when grown in glucose with HBA and VA, but less than $20 \%$ when grown in HBA and VA alone. When grown in phenol and glucose, $R$. rhodochrous accumulated $35 \%$ of its dry weight at lipids, but did not accumulate lipids when grown in phenol alone. These data indicate that $R$. rhodochrous may have the capability to tolerate and utilize lignin-like aromatic compounds for lipid accumulation.
\end{abstract}




\subsection{Introduction}

Lignocellulosic biomass (i.e. agricultural residues, wood, grasses, and energy crops) is one of the most abundant organic materials in the world, gaining interest from sustainable energy researchers to be used for high-value products. Lignocellulosic material is comprised of cellulose, hemicellulose, and lignin, which are chemically bonded and intricately intertwined. Several pretreatment methods have been developed to depolymerize lignocellulose and release the glucose and pentose sugars, mainly xylose, from the cellulose and hemicellulose, respectively (Hu and Ragauskas, 2012; Mosier et al., 2005). As a result of lignocellulosic pretreatment, inhibitors such as acids, furans, and phenolic compounds form in lignocellulosic hydrolysate, which can affect fermentation productivity (Palmqvist and Hahn-Hagerdal, 2000). While cellulose and hemicellulose can be used to produce various bioproducts, lignin is a complex polyaromatic structure that is recalcitrant to most bacteria and therefore limited in its use as a renewable and valuable resource.

Lignin accounts for $10-25 \%$ of lignocellulosic material and the global paper industry alone produces almost 50 million tons of lignin per year (Gosselink et al., 2004). Only $2 \%$ of lignin is used commercially, while the remainder is usually burned for energy. However, bioconversion of lignin offers exceptional potential for the microbial production of alternative fuels or bioproducts (Kosa and Ragauskas, 2012; Zakzeski et al., 2010). During the pretreatment of lignocellulosic material to release sugars for fermentation purposes, degradation of lignin to lignin oligomers and phenolic 
derivatives, such as vanillic acid, trans-p-coumaric acid and 4-hyroxybenzoic acid also occurs (Palmqvist and Hahn-Hagerdal, 2000; Saadia and Ashfaq, 2010). Degraded lignin fractions are often discarded due to lack of use and are ultimately found in industrial wastewaters (Lamichhane Upadhyaya et al., 2013; Saadia and Ashfaq, 2010; Wells et al., 2015).

Microbial degradation of lignin is limited to white-rot and brown-rot fungi, which are able to mineralize lignin, and to some soil bacteria, which are able to depolymerize lignin or further break-down lignin fragments (Bugg et al., 2011; Gelbrich et al., 2008). Several species of soil bacteria capable of aromatic degradation are currently being investigated for lignin degrading abilities, and these are focused on identification of specific enzymes and catabolic pathways (Bugg et al., 2011). Biodegradation of phenolic contaminants by microorganisms has been analyzed extensively. For instance, several Rhodococcus species have been shown to degrade phenol and other aromatic hydrocarbons (Larkin et al., 2005; Martinkova et al., 2009). Rhodococcus phenolicus sp. nov. was isolated from a wastewater bioprocessor and was shown to grown in up to $7.5 \mathrm{~g} / \mathrm{L}$ phenol as the sole carbon source (Rehfuss and Urban, 2005). Rhodococcus opacus GM-14 was also shown to use up to $1.2 \mathrm{~g} / \mathrm{L}$ of phenol and its halogenated derivatives as a sole carbon source (Zaitsev et al., 1995).

Phenol and its derivatives can be serious health hazards if released into the environment and aerobic degradation of phenolic compounds by bacteria, such as Rhodococcus species, is an important method of remediation (Buchholz and Pawliszyn, 1993; 
Michalowicz and Duda, 2007). The process of phenol degradation begins with the formation of catechol from the aromatic ring by phenol hydroxylase. R. erythropolis was genetically modified with phenol catabolism genes, including phenol hydroxylase and catechol 1,2-dioxygenase, suggesting that phenol degradation can be improved in Rhodococcus(Zídková et al., 2013). Catechol 1,2-dioxygenase, an ortho-cleaving enzyme, or catechol 2,3-dioxygenase, a meta-cleaving enzyme, are proteins involved in the Beta-ketoadipate pathway in which the bacterial degradation of catechol to central metabolic intermediates occurs (Guzik et al., 2011; Szokol et al., 2014; Zídková et al., 2013). The Beta-ketoadipate pathway converts catechol, derived from simple aromatics like phenol, or protocatechuate, derived from aromatic lignin monomers, to succinyl-CoA and Acetyl-CoA. Acetyl Co-A is necessary precursor for the fatty acid synthesis and lipid accumulation in Rhodococcus (Wynn and Ratledge, 2005). Phenol degraded via phenol hydroxylase and the Beta-ketoadipate pathway can generate acetyl-CoA for lipid accumulation by Rhodococcus species (Wells and Ragauskas, 2012) and this study aims to further elucidate this phenomenon using $R$. rhodochrous.

Another pathway involved in fatty acid synthesis in actinomycetes is the Beta-oxidation pathway, which has been shown to degrade hydrocarbons, including those with alkyl side chains, and is capable of producing membrane lipids and triglycerides (Alvarez, 2003). The Beta-oxidation pathway is active in normal growth conditions and in nutrientrestricting conditions, such as those present in the natural environments of actinomycetes, suggesting that the routine hydrocarbon degradation activity of actinomycetes can be used for growth or for fatty acids (Alvarez, 2003). The incorporation of hydrocarbons 
into cellular lipids, causing uncommon fatty acids (in regard to the substrate) has been observed in several species of Rhodococcus (Alvarez et al., 1996; Alvarez and Steinbuchel, 2002) and is explained by the key role of the Beta-oxidation pathway in fatty acid synthesis in actinomycetes (Alvarez, 2003).

Oleaginous microbes, which are those that can accumulate over $20 \%$ of their dry cellular weight as lipids (Wynn and Ratledge, 2005), have been studied for their biodegradative properties. For instance, the well-characterized oleaginous bacterium R. opacus (Alvarez et al., 1996) was recently found to convert model lignin compounds to triglycerides (Kosa and Ragauskas, 2012) and tolerate lignocellulosic hydrolysates during growth and lipid accumulation (Wang et al., 2014; Wells et al., 2015). Rhodococcus rhodochrous, which is known for its degradation capabilities, was recently identified as an oleaginous bacterium when grown in glucose (Shields-Menard et al., 2015). In an effort to better understand the mechanisms of aromatic degradation as it promotes lipid accumulation, the purpose of this study was to evaluate the effects of model lignin aromatic compounds, specifically phenol, vanillic acid (VA), and 4-hydroxybenzoic acid (HBA) on growth and lipid accumulation of $R$. rhodochrous.

\subsection{Materials and Methods}

\subsection{Culture and Experimental conditions}


R. rhodochrous (ATCC \#21198) glycerol stocks were stored at $-80^{\circ} \mathrm{C}$ and used to inoculate nutrient agar plates (Fisher Scientific, Pittsburg, Pennsylvania). Lawns of $R$. rhodochrous were used to inoculate experimental starters.

Shake-flask experiments were used to evaluate the growth and lipid accumulation by $R$. rhodochrous when grown in media supplemented with model lignin compounds. For phenol experiments, a minimal salts medium (Sorkhoh et al., 1990) was supplemented with $20 \mathrm{~g} / \mathrm{L}$ glucose, $1 \mathrm{~g} / \mathrm{L}$ phenol, or $20 \mathrm{~g} / \mathrm{L}$ glucose and $1 \mathrm{~g} / \mathrm{L}$ phenol. Bacterial inocula $(10 \% \mathrm{v} / \mathrm{v})$ for phenol experiments were prepared using a starter culture of $R$. rhodochrous grown in glucose-supplemented medium for 48 hours. The shake flask experiments were conducted in triplicates and the experimental mean and standard deviation were calculated for all gravimetric and sugar analyses. Repeated measures ANOVA were performed using the Statistical Package for the Social Sciences (SPSS) software v23 when necessary and significance was determined when $\mathrm{p}<0.05$.

For HBA and VA shake-flask experiments, Sorkhoh media (Sorkhoh et al., 1990) was supplemented with or without glucose $(20 \mathrm{~g} / \mathrm{L})$ and with $1.25 \mathrm{~g} / \mathrm{L}$ VA, $1.25 \mathrm{~g} / \mathrm{L} \mathrm{HBA}$, or both $1.25 \mathrm{~g} / \mathrm{L}$ HBA and $1.25 \mathrm{~g} / \mathrm{L}$ VA. A $1.25 \mathrm{~g} / \mathrm{L}$ concentration of VA or HBA was used based on previous literature (Kosa and Ragauskas, 2012) as well as previous observations (unpublished) of reduced growth and chemical solubility at higher concentrations. Inocula $(10 \% \mathrm{v} / \mathrm{v})$ were prepared for each treatment using the respective 48 -hour starter cultures of $R$. rhodochrous grown in glucose and VA, glucose and HBA, or glucose, HBA, and VA. The $\mathrm{pH}$ was adjusted with $\mathrm{NaOH}$ to 6.8 prior to inoculation. All cultures were incubated at $30^{\circ} \mathrm{C}$ in a shaking incubator at $125 \mathrm{rpm}$ (New Brunswick Scientific 
Model I26, Edison, New Jersey). All shake-flask experiments were conducted in triplicates and the experimental mean and standard deviation were calculated for all gravimetric and sugar analyses.

BIOFLO 310 5L fermenters (New Brunswick Scientific, Edison, NJ) with a 3L working volume were used to further evaluate the effects on growth and lipid accumulation of VA or HBA with glucose, in comparison to glucose only controls. The $\mathrm{pH}$ of all cultures was adjusted to 7 using $\mathrm{NaOH}$ and monitored (but not controlled) using the fermenters' $\mathrm{pH}$ probes. Temperature was maintained at $30^{\circ} \mathrm{C}$ and fermentation parameters were set as previously described (Shields-Menard et al., 2015). Samples were collected as described below every 12 hours until 48 hours and then daily until 168 hours.

\subsection{Analyses}

Culture samples $(50 \mathrm{~mL})$ were collected daily from all experiments. Samples were centrifuged at $2825 \mathrm{x} \mathrm{g}$ for 20 minutes and the supernatant was transferred to another vial for $\mathrm{pH}$ (Accumet), glucose, HBA, VA, and phenol analysis. The pellets were stored at $20^{\circ} \mathrm{C}$ and then freeze-dried (Freezone). Cell dry weight (CDW) was determined by subtracting the initial mass of the sample tube from final mass of the tube containing the freeze-dried cell pellet. Glucose concentration was measured for all samples in triplicate using a YSI 2900 Biochemistry Analyzer (YSI Incorporated Life Sciences, Yellow Springs, $\mathrm{OH})$. 


\subsection{Lipid Analysis}

Lipids were extracted from the cell pellet using a modified Bligh and Dyer method (Bligh and Dyer, 1959), with minor modifications as described previously (Revellame et al., 2012; Shields-Menard et al., 2015). Solvent was evaporated using a TurboVap LV (Caliper Life Sciences, Hopkinton, MA, USA) at $50^{\circ} \mathrm{C}$ with a 15 psi stream of nitrogen gas for 50 minutes before the extracted lipids were weighed to determine the gravimetric lipid accumulation. The extracted lipids were then transesterified into fatty acid methyl esters (FAMEs) and analyzed by three injections of each sample (Revellame et al., 2012). An Agilent 6890N gas chromatograph with a flame-ionization detector (GC-FID; Agilent Technologies Inc., Wilmington, DE, USA) and a Zebron ZB-FFAP column (30 m x 0.25 $\mathrm{mm}$, film thickness $0.25 \mu \mathrm{m}$ ) was calibrated using a standard solution of C9-C24 FAMEs in known concentrations (Sigma Aldrich, St. Louis, MO, USA) with 1, 3dichlorobenzene as an internal standard. Helium at a flow rate of $1.5 \mathrm{~mL}$ per minute was used as the carrier gas. The detector temperature was $260^{\circ} \mathrm{C}$ and the oven temperature was $50-250^{\circ} \mathrm{C}$ with a rate of $10^{\circ} \mathrm{C} /$ minute.

\subsection{Phenol, HBA, and VA Analysis}

An Agilent 6890N GC-FID (Agilent Technologies Inc., Wilmington, DE, USA) equipped with a solid-phase microextraction fiber (SPME; Sigma Aldrich, St. Louis, MO, USA) was used to determine phenol concentration as it avoids loss of analytes compared to other methods (Buchholz and Pawliszyn, 1993). The SPME was set for a vial penetration 
of $25 \mathrm{~mm}$ and an extraction time of 30 seconds followed by desorbing to the injector for 30 seconds. Injection penetration was $50 \mathrm{~mm}$. The injector was maintained at $280^{\circ} \mathrm{C}$ and split in a ratio of $10: 1$ and the detector was set at $300^{\circ} \mathrm{C}$. The oven temperature was programmed to $80^{\circ} \mathrm{C}$ for 2 minutes with an increase of $10^{\circ} \mathrm{C} /$ minute to $150^{\circ} \mathrm{C}$ for 9 minutes and an increase of $20^{\circ} \mathrm{C} /$ minute to $300^{\circ} \mathrm{C}$ for 17 minutes. A Phenomenex $\mathrm{ZB}-5$ $5 \%$ phenyl capillary column (30 m x $250 \mu \mathrm{m} \times 0.25 \mu \mathrm{m})$ was calibrated using known concentrations of phenol.

To monitor HBA and VA concentrations, samples were collected every two hours from BIOFLO 310 fermenters. Samples were filtered using a $0.45 \mu \mathrm{m}$ Teflon filter. Analysis was performed on an Agilent 1260 Infinity Liquid Chromatography (LC) system coupled to Agilent 380 evaporative light scattering detector (Santa Clara, CA) using a modified method (Verette). The chromatography separation was achieved with a Poroshell 120 EC-C18 column $(3.0 \times 150 \mathrm{~mm}, 2.7 \mu \mathrm{m})$ preceded by a Poroshell 120 EC-C18 guard column (2.1 x $5 \mathrm{~mm}, 2.7 \mu \mathrm{m})$, both from Agilent (Santa Clara, CA, USA). The mobile phase consisted of: A $\left(99.5 \% \mathrm{H}_{2} \mathrm{O} / 0.5 \%\right.$ formic acid v/v) and $\mathrm{B}(99.5 \%$ acetonitrile/0.5\% formic acid v/v). Formic acid (>99.5\%) and liquid chromatography grade acetonitrile and water were obtained from Fisher Scientific (Bridgewater, NJ, USA). Flow rate was set at $0.6 \mathrm{~mL} / \mathrm{minute}$ with an injection volume of $10 \mu \mathrm{L}$. The gradient was initially held at $5 \%$ B for 2 minutes. There was a linear increase from 2 to 9 minutes $(12 \% \mathrm{~B})$, from 9 to 11 minutes $(15 \% \mathrm{~B})$, from 11 to 14 minutes $(50 \% \mathrm{~B})$, and a linear decrease from 14 to 15 minutes $(5 \% \mathrm{~B})$. The equilibration was achieved 4 minutes after returning to the initial conditions. Identification and quantification were carried out using the evaporative light 
scattering detector (ELSD). ELSD parameters included nebulization with nitrogen at $28^{\circ} \mathrm{C}$ and a flow rate of 2.75 standard L per minute (SLM). The evaporator temperature was $30^{\circ} \mathrm{C}$.

\subsection{Protein Analysis}

Culture protein lysates were prepared using a modified Rhodococcus protein extraction method (Patrauchan et al., 2005). Briefly, cell pellets were disrupted by bead-beating in 10:1 v/v cell pellet to lysis buffer ( $8 \mathrm{M}$ urea, $4 \%$ CHAPS, $30 \mathrm{mM}$ Tris $\mathrm{pH} 8.5$, and protease inhibitor cocktail (Sigma P2714). Zirconia/silica beads ( $0.5 \mathrm{~g}$ of $0.1 \mathrm{~mm}$ beads) were used for bead-beating for 5 cycles of 1 minute and cooled on ice between cycles. Debris was removed by centrifugation at $34,180 \times \mathrm{g}$ for 30 minutes at $10^{\circ} \mathrm{C}$, discarding the pellet, and centrifuging the supernatant at $16,100 \mathrm{x} g$ at $10^{\circ} \mathrm{C}$ for 10 minutes. Protein concentration was quantified using a Fluka Protein Quantification Kit and equal amounts (100ug) of protein from each sample was precipitated using methanol and chloroform (4:1). Protein pellets were resuspended in $100 \mathrm{mM}$ ammonium bicarbonate and 5\% acetonitrile for trypsin digestion. Dithiothreitol (DTT) $(50 \mathrm{mM})$ was added for 10 minutes and proteins were incubated at $65^{\circ} \mathrm{C}$ followed by the addition of $100 \mathrm{mM}$ Iodoacetamide (IAA) for 30 minutes in a dark incubator at $30^{\circ} \mathrm{C}$. Trypsin $(0.1 \mu \mathrm{g} / \mu \mathrm{l}$; Pierce Trypsin Protease, Mass Spectrometry- grade, Thermo Scientific) was added and proteins were digested overnight at $37^{\circ} \mathrm{C}$. Glacial acetic acid was used to stop the digestion and detergents and salts were removed by using a Pierce Detergent Removal Spin Column (Thermo Scientific) and a macrotrap (Michrome Bioresources), both by 
using manufacturer's instructions. The samples were dried by vacuum centrifuge at room temperature. Desalted samples were resuspended in $0.1 \%$ formic acid with $5 \%$ acetonitrile for analysis.

Peptide mass spectrometry was accomplished using a Dionex UltiMate 3000 (Thermo Scientific) high performance liquid chromatography machine (HPLC) coupled with an LTQ-OrbiTrap Velos (Thermo Scientific) tandem mass spectrometer. The Dionex UltiMate 3000 was configured for reversed phase chromatography using an Acclaim PepMap RSLC column (Thermo Scientific) with a flow rate of $300 \mathrm{~nL} /$ minute. Peptides were separated for mass spectrometry analysis using an acetonitrile gradient starting at $2 \% \mathrm{ACN}, 0.1 \% \mathrm{FA}$ and reaching $50 \% \mathrm{ACN}, 0.1 \% \mathrm{FA}$ in 120 minutes, followed by a 15 minutes wash of $95 \%$ ACN, $0.1 \%$ FA. Column equilibration was handled automatically using the Dionex UltiMate 3000. The eluate from the HPLC was fed directly to the LTQOrbiTrap Velos for nanospray ionization followed by MS/MS analysis of detected peptides. The LTQ-OrbiTrap Velos was configured to perform $1 \mathrm{~ms}$ scan followed by 18 MS/MS scans of the 18 most intense peaks repeatedly over the 135 min duration of each HPLC run.

Tandem mass spectra (MS/MS) were extracted and the charge state was deconvoluted and deisotoped by Proteome Discoverer (Proteome Discoverer ver. 1.4.0.288, Thermo Fisher Scientific, CA, USA). The analysis was performed by using Sequest (XCorr) (Thermo Fisher Scientific, CA, USA) and X! Tandem (version CYCLONE 2010.12.01.1). The NCBI database for Rhodococcus rhodochrous ATCC (version from 
March 2015, 6034 entries) was used for the proteomic comparisons, using the in silico trypsin digestion, with a fragment ion mass tolerance of $0.80 \mathrm{Da}$ and a parent ion tolerance of 1.5 Da. Oxidation of methionine and carbamidomethylation of cysteine were chosen as variable modifications in both searches. Additionally, Glu->pyro-Glu of the Nterminus, ammonia-loss of the N-terminus, and gln->pyro-Glu of the N-terminus were specified in X! Tandem as variable modifications. Scaffold (version 4.4.5, Proteome Software Inc., Portland, OR, USA) was used to validate MS/MS based peptide and protein identifications. Peptide identifications were accepted if they achieved a false delivery rate (FDR) less than $0.1 \%$. Peptide probabilities from $\mathrm{X}$ ! Tandem were assigned by the Peptide Prophet algorithm (Keller et al., 2002) with Scaffold delta-mass correction. Peptide probabilities from Sequest (XCorr Only) were assigned by the Scaffold Local FDR algorithm. Protein identifications were accepted if they could be established at greater than $99.0 \%$ probability and contained at least 2 identified peptides. Protein probabilities were assigned by the Protein Prophet algorithm (Nesvizhskii et al., 2003). Proteins that contained similar peptides and could not be differentiated based on MS/MS analysis alone were grouped to satisfy the principles of parsimony. Fold change was calculated using weighted spectral counts and a minimum value of 0.5 counts was used for the proteins with a spectral count of 0 in order to calculate the fold change; minimum 1.5 fold change was required for differential expression analysis. The statistical analysis was performed using Fisher's test with Hochberg-Benjamini correction (see pvalues in Table 2).

\subsection{Results and Discussion}




\subsection{Phenol reduces, but does not inhibit growth and lipid accumulation of $R$. rhodochrous}

Phenol was used to investigate the effects of a common aromatic compound present in lignocellulosic hydrolysates on the growth and lipid accumulation of $R$. rhodochrous. Cell dry weight of $R$. rhodochrous increased to $4 \mathrm{~g} / \mathrm{L}$ after 120 hours of growth in glucose and phenol supplemented media, although overall growth in the mixture of glucose and phenol was significantly less than the glucose control (Figure 1A and 1C). Phenol concentrations were below the level of detection by GC-FID at 48 hours in both the phenol and phenol with glucose treatments (Figure 1A), but the phenol concentration in the abiotic control remained close to $1 \mathrm{~g} / \mathrm{L}$, suggesting that $R$. rhodochrous utilized phenol as a carbon source for growth. The increase in cell dry weight of $R$. rhodochrous grown in phenol as the sole carbon source is significantly less compared to that of the glucose control and combination of glucose and phenol; however, when comparing the cell dry weight at 120 hours to time 0 , there is a noticeable increase (Figure 1A and 1C), further suggesting that $R$. rhodochrous can tolerate and utilize $1 \mathrm{~g} / \mathrm{L}$ of phenol as a carbon source. Phenol, a common ingredient in cleaning supplies, is generally considered toxic to most microbes and has been shown to have inhibitory effects on growth and yields of fermentative organisms (Klinke et al., 2004; Palmqvist and Hahn-Hagerdal, 2000). Rhodococcus species have been well described for their metabolic capabilities in degrading a plethora of compounds (Larkin et al., 2005; Martinkova et al., 2009). In this study, $R$. rhodochrous could tolerate and use $1 \mathrm{~g} / \mathrm{L}$ phenol as a sole carbon source for growth, but achieved greater growth when the media was supplemented with glucose. Similarly, Pseudomonas putida overcame the inhibitory effect of phenol when cultivated 
with glucose as an added growth substrate (Mamma et al., 2004). A recent study further suggests that a key enzyme of the phenol degradation pathway, phenol hydroxylase was repressed by succinate, but not by the addition of glucose (Szokol et al., 2014; Zídková et al., 2013). While the data shows the degradation of phenol and growth of $R$. rhodochrous using both glucose and phenol, the slower growth rate might indicate the presence of another catabolite repressing an enzyme involved in phenol degradation.

\subsection{Lipid analysis and FAME profiles of $R$. rhodochrous grown in phenol}

Lipid accumulation by $R$. rhodochrous grown in a mixture of glucose and phenol was evident within 48 hours, though glucose only controls began accumulating within the first 24 hours (Figure 1 B and 1D). R. rhodochrous accumulated over 30\% of cell dry weight as lipids when grown in phenol with glucose, which is still considered oleaginous (over $20 \%$ ) even though the glucose control had a significantly greater yield of $60 \%$ lipid (Figure 1B). When R. rhodochrous was grown in just phenol, lipid content was significantly lower than both glucose control and glucose and phenol treatment and lipid content decreased to $0.03 \mathrm{~g} / \mathrm{L}$ ( $3 \%$ of $\mathrm{CDW}$ ) by 120 hours (Figures $1 \mathrm{~B}$ and $1 \mathrm{D}$ ), suggesting that these lipids were consumed for growth after phenol exhaustion at 48 hours (Figure 1C and 1D). Lipid accumulation by R. rhodochrous grown in phenol as a sole carbon source was likely limited due to the lack of available carbon $(1 \mathrm{~g} / \mathrm{L}$ phenol $)$ in the media. Further studies should aim to increase the amount of phenol available to $R$. rhodochrous to promote lipid accumulation without having toxic effects. 
Low molecular weight phenolic compounds have been shown to be more toxic than larger phenolics, inhibiting microbial growth and fermentation by causing membrane instability (Klinke et al., 2004; Palmqvist and Hahn-Hagerdal, 2000). A previous study (Tsitko et al., 1999) investigated the effects of aromatic compounds on the fatty acid composition of $R$. opacus and found that cells grown on aromatic substrates had increased amounts of odd-chain fatty acids. Similarly, the data indicated an increase in unknown FAMEs at 72 hours (Table 1), which corresponded to an unidentifiable peak with a retention time between that of C16:0 and C18:1. If these unknown FAMEs are indeed odd-chain saturated fatty acids, then an increase in saturated fatty acids suggests that $R$. rhodochrous may be reacting to the presence of membrane-active compounds and has a protection mechanism against cell disruption by aromatic compounds (Tsitko et al., 1999). Changes in FAME profiles have also been observed in Pseudomonas species during phenol contaminated soil studies between 40-60 days suggesting that FAME analysis may be a good tool to monitor phenol bioremediation efforts (Mrozik et al., 2010; Mrozik et al., 2008).

\subsection{Proteomic analysis of $R$. rhodochrous grown in phenol}

Almost 500 proteins from each treatment were identified in the proteomic analysis, several of which were related to aromatic degradation, central metabolism, fatty acid metabolism, nitrogen metabolism, and other important cellular functions (Table 2). The mechanism of lipid synthesis and accumulation in yeasts is generally well understood and provides the mechanistic foundation for lipid accumulation in oleaginous microbes (Jin et al., 2015). Recent proteomic comparisons have gained valuable insight towards the 
triacylglyercol (TAG) biosynthesis, storage, and degradation among oleaginous

organisms, including Rhodococcus (Chen et al., 2014; Davila Costa et al., 2015).

When cultured in glucose, $R$. rhodochrous accumulated 25\% of CDW as lipid within 24 hours (Figures 1B and D). As lipid accumulation is beginning, there is also a large increase in non-lipid biomass (growth) between 0 and 24 hours as nitrogen is not yet limited (previous data). When the protein abundance at 24 hours was compared to that at 0 hours, there was a 25 -fold increase in biotin carboxylase (oxaloacetate decarboxylase), an enzyme subunit of acetyl-CoA carboxylase (ACC) responsible for shunting the flux of carbon to lipids by catalyzing the conversion of acetyl-CoA to malonyl-CoA for fatty acid synthesis (Davila Costa et al., 2015; Magdouli et al., 2014). The switch from cell growth (oxidative catabolism) to lipid accumulation (reductive anabolism) was also evident by the increased expression of acyltransferase, citrate synthase and 3isopropylmalate dehydrogenase and the decrease in abundance of adenylate kinase (Table 2). Malic enzyme, which is critical in replenishing NADPH during fatty acid synthesis in nitrogen starvation, had a 16.7-fold reduction within 24 hours (Table 2). The abundance of proteins involved in both lipid synthesis and cell growth suggest that $R$. rhodochrous is likely entering limited nitrogen conditions, slowing cell growth, and beginning lipid accumulation.

Phenol was undetected in the media by 48 hours, suggesting that $R$. rhodochrous was able to degrade and use phenol as a carbon source (Figure 1A). Several proteins involved in aromatic degradation (Guzik et al., 2011; Mamma et al., 2004; Prieto et al., 1993; 
Wells and Ragauskas, 2012; Zídková et al., 2013) were in high abundance in phenol cultures at 24 hours compared to 0 hour: multiple putative $\mathrm{HpaB} / \mathrm{PvcC} / 4-\mathrm{BUDH}$ (phenol hydroxylase); catechol 1, 2-dioxygenases; and catechol 2, 3-dioxygenase (Table 2). Proteins involved in central metabolism and fatty acid synthesis, such as 6phosphogluconate dehydratase, an amino acid transport system, propionyl-CoA carboxylase and acyltransferase, were less abundant at 24 hours than 0 hour, which corresponds to the decrease of lipid accumulation at 24 hours. TadA $(2,3-$ bisphosphoglycerate-dependent phosphoglycerate mutase) is involved in the structural formation of lipid bodies (Ding et al., 2012; MacEachran et al., 2010) and was found to be increased by 11-fold at 24 hours. Biotin carboxylase and malic enzyme were also over 2 -fold higher at 24 hours than 0 hour. The presence of proteins involved in fatty acid synthesis may indicate that greater lipid accumulation is possible using phenol as a substrate if phenol can be used in larger concentrations.

The primary metabolic activities of $R$. rhodochrous grown in glucose or in phenol are evident when the abundance of proteins in each are compared at 24 hours. Catechol 2,3dioxygenase, catechol 1, 2-dioxygenase, and multiple putative $\mathrm{HpaB} / \mathrm{PvcC} / 4-\mathrm{BUDH}$ (phenol hydroxylase) are significantly less abundant, which shows the difference in activity of phenol degradation. In comparison, propionyl-CoA carboxylase and acyltransferase were 78- and 44-fold higher in $R$. rhodochrous grown in glucose at 24 hours than in phenol at 24 hours, further supporting the increased lipid accumulation observed in glucose grown cells at 24 hours. 
When $R$. rhodochrous was grown in a combination of glucose and phenol, there was a slower increase in lipid accumulation. The proteomic analysis of $R$. rhodochrous grown in glucose and phenol indicated an increase at 24 hours in proteins associated with phenol degradation, such as catechol 1, 2-dioxygenase and a putative HpaB/PvcC/4-BUDH enzyme (phenol hydroxylase), as well as those proteins associated with lipid synthesis, 3hydroxybutyryl-CoA epimerase and biotin carboxylase. Proteins associated with nitrogen scavenging, ABC-type branched-chain amino acid transport systems periplasmic component-like protein and a urea $\mathrm{ABC}$ transporter, had the highest negative fold change compared to that at 0 hours. Similarly, when the proteins identified in glucose and phenol at 24 hours were compared to those in glucose at 24 hours, phenol degradation proteins and lipid synthesis proteins were more prevalent, further supporting that the metabolic diversity of $R$. rhodochrous is capable of concurrent aromatic degradation and lipid accumulation. Further research is needed to identify the specific roles and regulatory components of $R$. rhodochrous.

3.4 VA and HBA do not inhibit growth or lipid accumulation when R. rhodochrous is grown in the presence of glucose

VA and HBA are major aromatic components in untreated hydrolysate (Palmqvist and Hahn-Hagerdal, 2000) and can be used as model compounds representing the major components of grass, hardwood, and softwood lignin (Kasi and Ragauskas, 2010; Pu et al., 2011). R. rhodochrous was cultured in shake flasks with HBA or VA as a sole carbon 
source and supplemented with glucose to investigate the effects of these model aromatic lignin compounds on growth and lipid accumulation. After 72 hours of cultivation, growth was observed to be greater when $R$. rhodochrous was supplemented with glucose than those with HBA and VA as a sole carbon source (Figure 2A). Furthermore, percent lipid accumulation was much greater in the cultures supplemented with glucose than those without (Figure 2 B).

The utilization of HBA and VA by $R$. rhodochrous was also analyzed using BIOFLO 310 fermenters; concentrations of HBA and VA were measured every 2 hours for 24 hours. The data indicated that HBA and VA were undetected by 18 hours and 20 hours, respectively (Figure 3D). This suggests that the low CDW and lipid accumulation analyzed at 72 hours was due to an exhaustion of carbon. However, when $R$. rhodochrous was cultured with glucose in the presence of VA and HBA, CDW increased to over $5 \mathrm{~g} / \mathrm{L}$, only slightly less than the glucose control (Figure 3A). Lipid accumulation of over 40\% was also similar between cells grown in the presence of VA and HBA and that of the glucose control (Figure 3B), implying that model aromatic lignin compounds do not inhibit the growth or lipid accumulation of $R$. rhodochrous.

Since HBA and VA were undetected by 20 hours of cultivation, it was expected that lipid accumulation and cell growth would be comparable between treatments, assuming inhibitory effects were not present from 0-20 hours. The FAME analysis showed slight differences between the glucose control and the model lignin compound treatments in euric acid at time 0 and an increase of unknowns, likely odd chain carbon fatty acids, at 
24 hours (Figure 4). The slight differences in FAMEs may indicate a fatty acid response to aromatic compounds as observed when $R$. rhodochrous was cultivated in phenol. Furthermore, increasing $\mathrm{pH}$ (Figure 3C) could positively impact membrane transport, influencing the cell growth and lipid accumulation as proposed in previous work using the model oleaginous bacterium, R. opacus PD630 (Kosa and Ragauskas, 2012).

\subsection{Conclusions}

Rhodococcus rhodochrous is a versatile bacterium with a diverse metabolism capable of degradation and lipid accumulation. The prevalence and potential of the lignin component of lignocellulosic biomass for bioconversion to a product remains elusive. The purpose for using phenolic compounds in this study is twofold: phenol and it derivatives can be environment pollutants and phenol also serves as a basic model lignin compound as lignin is a polyaromatic structure. Proteomic analysis provides insight into the concurrent aromatic substrate degradation and lipid accumulation by $R$. rhodochrous. When cultivated in model aromatic lignin compounds (phenol, HBA, VA) and supplemented with glucose, $R$. rhodochrous growth and lipid accumulation increased. Furthermore, phenol, HBA, and VA did not have any considerable negative effects on $R$. rhodochrous and were undetected by 24 hours. Further work is necessary to fully elucidate the impact of lignin compounds on $R$. rhodochrous, so that growth and lipid yields can be improved without the use of glucose as a substrate.

\section{Acknowledgements}


This research was funded by the United States Department of Energy, Office of Energy Efficiency and Renewable Energy (Grant No.: DE-FG36-06G086025). This publication was developed under STAR Fellowship Assistance Agreement no. 91761201-0 awarded by the U.S. Environmental Protection Agency (EPA). It has not been formally reviewed by EPA. The views expressed in this publication are solely those of Sara A. Shields-Menard, and EPA does not endorse any products or commercial services mentioned in this publication.

\section{CONFLICT OF INTEREST}

The authors declare that all experiments documented in this manuscript comply with the current laws of the U.S.A.

The authors declare that they have no conflict of interest. 


\section{FIGURES}

Figure 1. Phenol concentration (grey; $\mathrm{g} / \mathrm{L} \pm \mathrm{SD}$ ) and $R$. rhodochrous cell dry weight (black; g/L \pm SD; A), percent lipid ( \pm SD; B), non-lipid biomass (g/L $\pm S D ; C$ ), and lipid (g/L $\pm \mathrm{SD} ;$ D) of $R$. rhodochrous shake flask cultures supplemented with $20 \mathrm{~g} / \mathrm{L}$ glucose $(\bullet), 1 \mathrm{~g} / \mathrm{L}$ phenol $(\boldsymbol{\square})$, or $1 \mathrm{~g} / \mathrm{L}$ phenol and $20 \mathrm{~g} / \mathrm{L}$ glucose $(\bullet)$.

Figure 2. $R$. rhodochrous cell dry weight $(\mathbf{A} ; \mathrm{g} / \mathrm{L} \pm \mathrm{SD})$ and percent lipid accumulation (B; \pm SD) of shake flask cultures supplemented with VA, HBA, VA + HBA, with and without glucose. Data shown from the 72-hour time point.

Figure 3. $R$. rhodochrous cell dry weight $(\mathbf{A} ; \mathrm{g} / \mathrm{L})$, percent lipid accumulation $(\mathbf{B})$ and $\mathrm{pH}$ (C) of 3L fermentations supplemented with glucose $(\bullet)$ and $\mathrm{VA}(\boldsymbol{\bullet}), \mathrm{HBA}(\bullet)$, or VA + HBA (-). Vanillic acid and 4-hydroxybenzoic acid concentrations (D;g/L) were analyzed using HPLC-ELSD. Fermentation samples of VA ( $\bullet$ ), HBA (•) and VA + HBA (grey; घ, ) were taken every 2 hours and filtered before analysis.

Figure 4. Fatty acid methyl ester profile (\% w/w) of R. rhodochrous grown in glucose (Glu), vanillic acid (VA), 4-hydroxybenzoic acid (HBA), or a mixture of VA and HBA $(\mathrm{VA}+\mathrm{HBA})$ at 0,12 , and 24 hours. Percentages are an average of triplicate injections of all experimental treatment replicates. Standard deviation is shown by error bars. FAMEs less than $1 \%$ are not shown. 


\section{References}

Alvarez, H.M., 2003. Relationship between $\beta$-oxidation pathway and the hydrocarbon-degrading profile in actinomycetes bacteria. International Biodeterioration \& Biodegradation 52, 35-42.

Alvarez, H.M., Mayer, F., Fabritius, D., Steinbuchel, A., 1996. Formation of intracytoplasmic lipid inclusions by Rhodococcus opacus strain PD630. Archives of microbiology 165, 377-386.

Alvarez, H.M., Steinbuchel, A., 2002. Triacylglycerols in prokaryotic microorganisms. Applied microbiology and biotechnology 60, 367-376.

Bligh, E.G., Dyer, W.J., 1959. A Rapid Method of Total Lipid Extraction and Purification. Canadian Journal of Biochemistry and Physiology 37, 911-917.

Buchholz, K.D., Pawliszyn, J., 1993. Determination of phenols by solid-phase microextraction and gas chromatographic analysis. Environmental Science and Technology 27, 2844-2848.

Bugg, T.D., Ahmad, M., Hardiman, E.M., Singh, R., 2011. The emerging role for bacteria in lignin degradation and bio-product formation. Current opinion in biotechnology 22, 394-400.

Chen, Y., Ding, Y., Yang, L., Yu, J., Liu, G., Wang, X., Zhang, S., Yu, D., Song, L., Zhang, H., Zhang, C., Huo, L., Huo, C., Wang, Y., Du, Y., Zhang, H., Zhang, P., Na, H., Xu, S., Zhu, Y., Xie, Z., He, T., Zhang, Y., Wang, G., Fan, Z., Yang, F., Liu, H., Wang, X., Zhang, X., Zhang, M.Q., Li, Y., Steinbuchel, A., Fujimoto, T., Cichello, S., Yu, J., Liu, P., 2014. Integrated omics study delineates the dynamics of lipid droplets in Rhodococcus opacus PD630. Nucleic Acids Res 42, 1052-1064.

Davila Costa, J.S., Herrero, O.M., Alvarez, H.M., Leichert, L., 2015. Label-free and redox proteomic analyses of the triacylglycerol-accumulating Rhodococcus jostii RHA1. Microbiology 161, 593-610.

Ding, Y., Yang, L., Zhang, S., Wang, Y., Du, Y., Pu, J., Peng, G., Chen, Y., Zhang, H., Yu, J., Hang, H., Wu, P., Yang, F., Yang, H., Steinbuchel, A., Liu, P., 2012. Identification of the major functional proteins of prokaryotic lipid droplets. J Lipid Res 53, 399-411.

Gelbrich, J., Mai, C., Militz, H., 2008. Chemical changes in wood degraded by bacteria. International Biodeterioration \& Biodegradation 61, 24-32. 
Gosselink, R.J.A., de Jong, E., Guran, B., Abächerli, A., 2004. Co-ordination network for lignin - standardisation, production and applications adapted to market requirements (EUROLIGNIN). Industrial Crops and Products 20, 121-129.

Guzik, U., Greń, I., Hupert-Kocurek, K., Wojcieszyńska, D., 2011. Catechol 1,2dioxygenase from the new aromatic compounds - Degrading Pseudomonas putida strain N6. International Biodeterioration \& Biodegradation 65, 504-512.

Hu, F., Ragauskas, A.J., 2012. Pretreatment and Lignocellulosic Chemistry. Bioenergy Research 5, 1043-1066.

Jin, M., Slininger, P.J., Dien, B.S., Waghmode, S., Moser, B.R., Orjuela, A., Sousa Lda, C., Balan, V., 2015. Microbial lipid-based lignocellulosic biorefinery: feasibility and challenges. Trends in biotechnology 33, 43-54.

Kasi, D., Ragauskas, A.J., 2010. Switchgrass as an energy crop for biofuel production: A review of its ligno-cellulosic chemical properties. Energy and Environmental Science 3, 1182-1190.

Keller, A., Nesvizhskii, A.I., Kolker, E., Aebersold, R., 2002. Empirical Statistical Model To Estimate the Accuracy of Peptide Identifications Made by MS/MS and Database Search. Analytical Chemistry 74, 5383-5392.

Klinke, H.B., Thomsen, A.B., Ahring, B.K., 2004. Inhibition of ethanol-producing yeast and bacteria by degradation products produced during pre-treatment of biomass. Applied microbiology and biotechnology 66, 10-26.

Kosa, M., Ragauskas, A.J., 2012. Bioconversion of lignin model compounds with oleaginous Rhodococci. Applied microbiology and biotechnology 93, 891-900.

Lamichhane Upadhyaya, K., Mondala, A., Hernandez, R., French, T., Green, M., McFarland, L., Holmes, W., 2013. Biocrude production by activated sludge microbial cultures using pulp and paper wastewaters as fermentation substrate. Environmental Technology 34, 2171-2178.

Larkin, M.J., Kulakov, L.A., Allen, C.C., 2005. Biodegradation and Rhodococcus-masters of catabolic versatility. Current opinion in biotechnology 16, 282-290.

MacEachran, D.P., Prophete, M.E., Sinskey, A.J., 2010. The Rhodococcus opacus PD630 heparin-binding hemagglutinin homolog TadA mediates lipid body formation. Applied and environmental microbiology 76, 7217-7225.

Magdouli, S., Yan, S., Tyagi, R.D., Surampalli, R.Y., 2014. Heterotrophic Microorganisms: A Promising Source for Biodiesel Production. Critical Reviews in Environmental Science and Technology 44, 416-454. 
Mamma, D., Kalogeris, E., Papadopoulos, N., Hatzinikolaou, D.G., Christrakopoulos, P., Kekos, D., 2004. Biodegradation of phenol by acclimatized Pseudomonas putida cells using glucose as an added growth substrate. Journal of Environmental Science and Health A39, 2093-2194.

Martinkova, L., Uhnakova, B., Patek, M., Nesvera, J., Kren, V., 2009. Biodegradation potential of the genus Rhodococcus. Environment international 35, 162-177. Michalowicz, J., Duda, W., 2007. Phenols - Sources and Toxicity. Polish Journal of Environmental Studies 16, 347-362.

Mosier, N., Wyman, C.E., Dale, B., Elander, R., Lee, Y.Y., Holtzapple, M.T., Ladisch, M., 2005. Features of promising technologies for pretreatment of lignocellulosic biomass. Bioresource technology 96, 673-686.

Mrozik, A., Cycoń, M., Piotrowska-Seget, Z., 2010. Changes of FAME profiles as a marker of phenol degradation in different soils inoculated with Pseudomonas sp. CF600. International Biodeterioration \& Biodegradation 64, 86-96.

Mrozik, A., Piotrowska-Seget, Z., Łabużek, S., 2008. FAMEs profiles of phenoldegrading Pseudomonas stutzeri introduced into soil. International Biodeterioration \& Biodegradation 62, 319-324.

Nesvizhskii, A.I., Keller, A., Kolker, E., Aebersold, R., 2003. A statistical model for identifying proteins by tandem mass spectrometry. Analytical Chemistry 75, 46464658.

Palmqvist, E., Hahn-Hagerdal, B., 2000. Fermentation of lignocellulosic hydrolysates. II: inhibitors and mechanisms of inhibition. Bioresource technology 74, 25-33.

Patrauchan, M.A., Florizone, C., Dosanjh, M., Mohn, W.W., Davies, J., Eltis, L.D., 2005. Catabolism of benzoate and phthalate in Rhodococcus sp. strain RHA1: redundancies and convergence. Journal of bacteriology 187, 4050-4063.

Prieto, M.A., Perez-Aranda, A., Garcia, J.L., 1993. Characterization of an Escherichia coli aromatic hydroxylase with a broad substrate range. Journal of bacteriology 175 , 2162-2167.

Pu, Y., Kosa, M., Kalluri, U.C., Tuskan, G.A., Ragauskas, A.J., 2011. Challenges of the utilization of wood polymers: how can they be overcome? Applied microbiology and biotechnology 91, 1525-1536.

Rehfuss, M., Urban, J., 2005. Rhodococcus phenolicus sp. nov., a novel bioprocessor isolated actinomycete with the ability to degrade chlorobenzene, dichlorobenzene and phenol as sole carbon sources. Systematic and applied microbiology 28, 695701. 
Revellame, E.D., Hernandez, R., French, W., Holmes, W.E., Benson, T.J., Pham, P.J., Forks, A., Callahan Ii, R., 2012. Lipid storage compounds in raw activated sludge microorganisms for biofuels and oleochemicals production. RSC Advances 2, 2015.

Saadia, A., Ashfaq, A., 2010. Environmental management in pulp and paper industry. Journal of INdustrial Pollution Control 26, 71-77.

Shields-Menard, S.A., Amirsadeghi, M., Sukhbaatar, B., Revellame, E., Hernandez, R., Donaldson, J.R., French, W.T., 2015. Lipid accumulation by Rhodococcus rhodochrous grown on glucose. Journal of industrial microbiology \& biotechnology 42, 693-699.

Sorkhoh, N.A., Ghannoum, M.A., Ibrahim, A.S., Stretton, R.J., Radwan, S.S., 1990. Sterols and diacylglycerosphocholines in the lipids of the hydrocarbon-utilizing prokaryote Rhodococcus rhodochrous. Journal of Applied Bacteriology 69, 856-863.

Szokol, J., Rucka, L., Simcikova, M., Halada, P., Nesvera, J., Patek, M., 2014. Induction and carbon catabolite repression of phenol degradation genes in Rhodococcus erythropolis and Rhodococcus jostii. Applied microbiology and biotechnology 98, 8267-8279.

Tsitko, I.V., Zaitsev, G.M., Lobanok, A.G., Salkinoja-Salonen, M.S., 1999. Effect of aromatic compounds on cellular fatty acid composition of Rhodococcus opacus. Applied and environmental microbiology 65, 853-855.

Verette, E., A new generation of evaporative light-scattering detectors for liquid chromatography: universality, reliability and cost-effectiveness in food analysis- an application review in HPLC and U-HPLC. SEDERE, http://www.sedere.com/wpcontent/uploads/SEDEX-LT-ELSD-Food-Analysis-Poster.pdf.

Wang, B., Rezenom, Y.H., Cho, K.C., Tran, J.L., Lee do, G., Russell, D.H., Gill, J.J., Young, R., Chu, K.H., 2014. Cultivation of lipid-producing bacteria with lignocellulosic biomass: effects of inhibitory compounds of lignocellulosic hydrolysates. Bioresource technology 161, 162-170.

Wells, T., Jr., Ragauskas, A.J., 2012. Biotechnological opportunities with the betaketoadipate pathway. Trends in biotechnology 30, 627-637.

Wells, T., Wei, Z., Ragauskas, A., 2015. Bioconversion of lignocellulosic pretreatment effluent via oleaginous Rhodococcus opacus DSM 1069. Biomass and Bioenergy 72, 200-205.

Wynn, J.P., Ratledge, C., 2005. Oils from Microorganisms, In: Shahidi, F. (Ed.), Bailey's Industrial Oil and Fat Products, 6 ed. John Wiley \& Sons, Inc., pp. 121-153. 
Zaitsev, G.M., Uotila, J.S., Tsitko, I.V., Lobanok, A.G., Salkinoja-Salonen, M.S., 1995. Utilization of halogenated benzenes, phenols, and benzoates by Rhodococcus opacus GM-14. Applied and environmental microbiology 61, 4191-4201.

Zakzeski, J., Bruijnincx, P.C.A., Jongerius, A.L., Weckhuysen, B.M., 2010. The Catalytic Valorization of Lignin for the Production of Renewable Chemicals. Chemical Reviews 110, 3552-3599.

Zídková, L., Szőköl, J., Rucká, L., Pátek, M., Nešvera, J., 2013. Biodegradation of phenol using recombinant plasmid-carrying Rhodococcus erythropolis strains. International Biodeterioration \& Biodegradation 84, 179-184. 
A

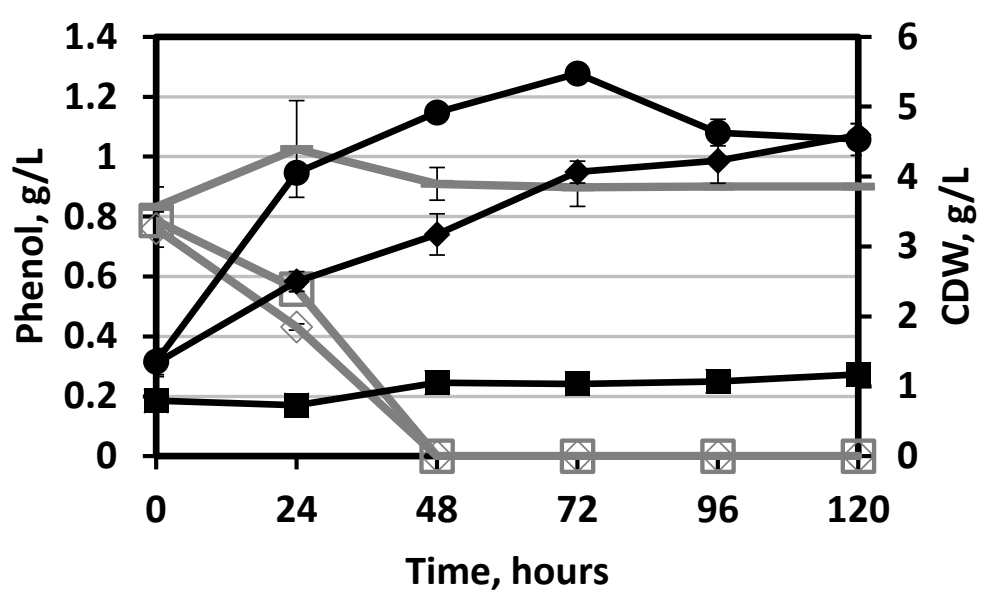

B

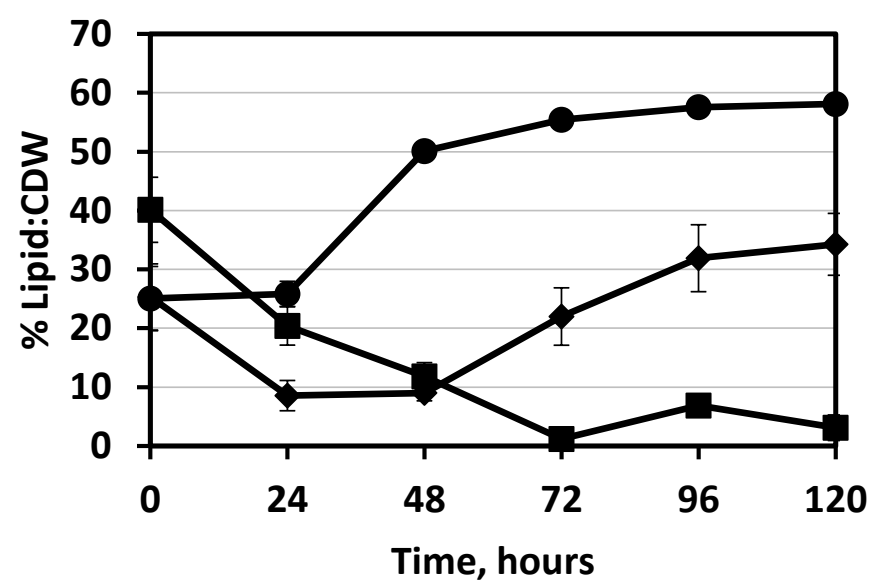

C

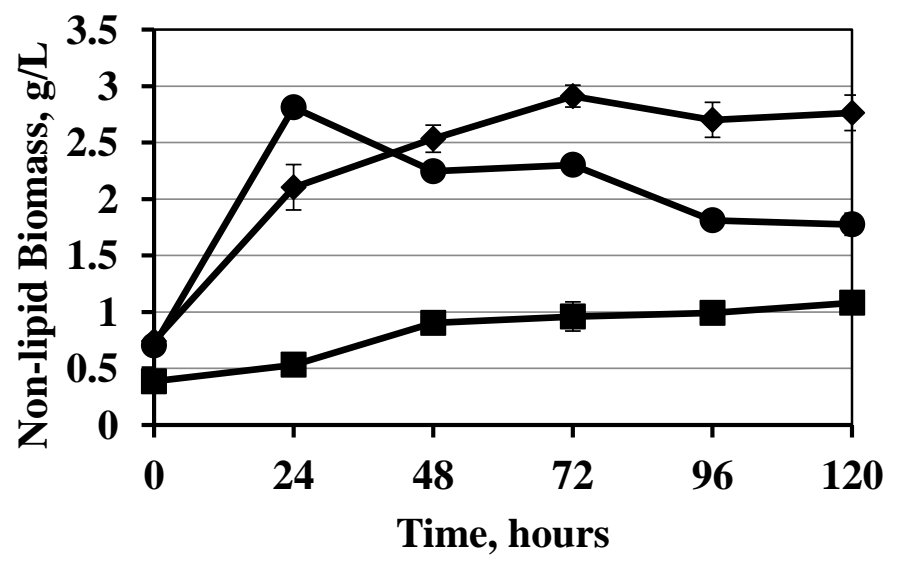


D

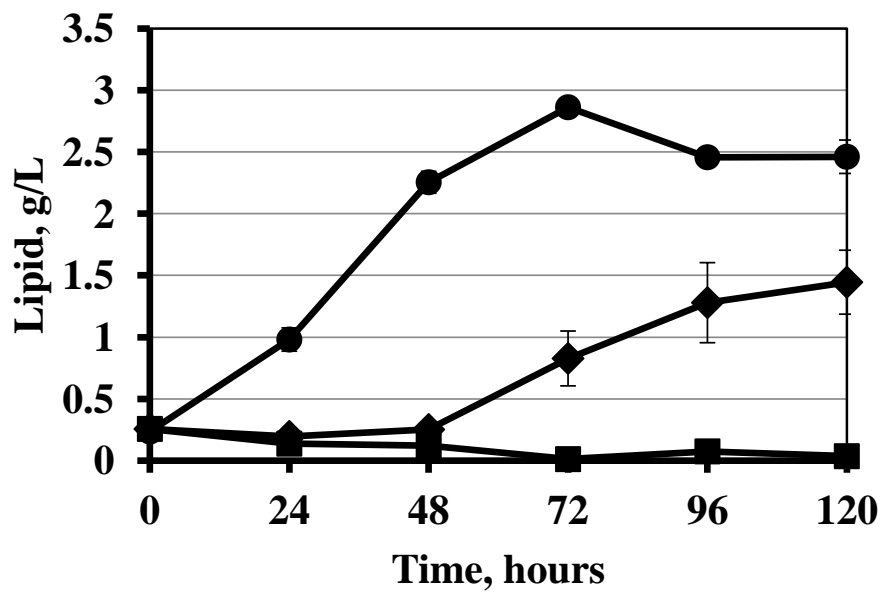

Fig. 1 Phenol concentration (grey; $g / L \pm S D$ ) and $R$. rhodochrous cell dry weight (black; g/L $\pm S D$;

A), percent lipid ( $\pm \mathrm{SD} ; \mathrm{B})$, non-lipid biomass (g/L $\pm \mathrm{SD} ; \mathrm{C})$, and lipid (g/L $\pm \mathrm{SD} ; \mathrm{D})$ of $R$. rhodochrous shake flask cultures supplemented with $20 \mathrm{~g} / \mathrm{L}$ glucose $(\bullet), 1 \mathrm{~g} / \mathrm{L}$ phenol $(\boldsymbol{\square})$, or $1 \mathrm{~g} / \mathrm{L}$ phenol and 20g/L glucose $(\bullet)$. 
A

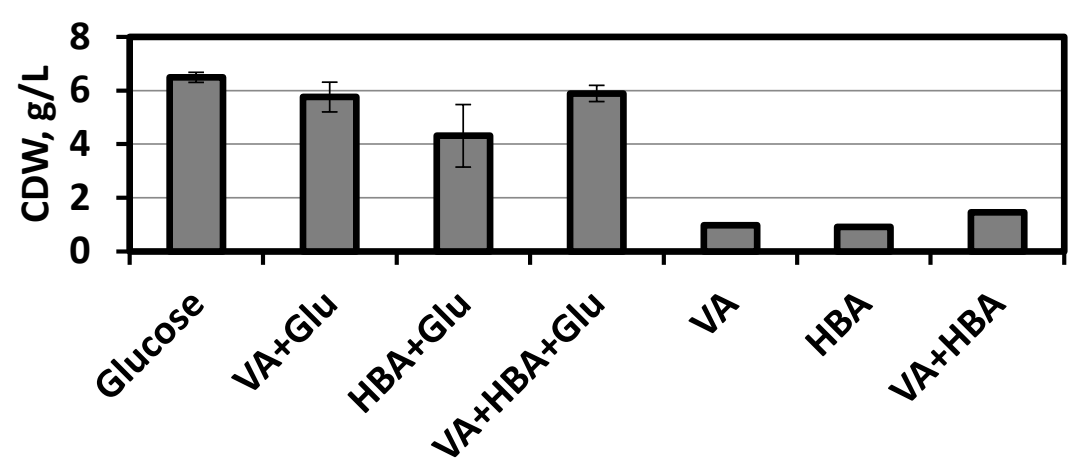

B

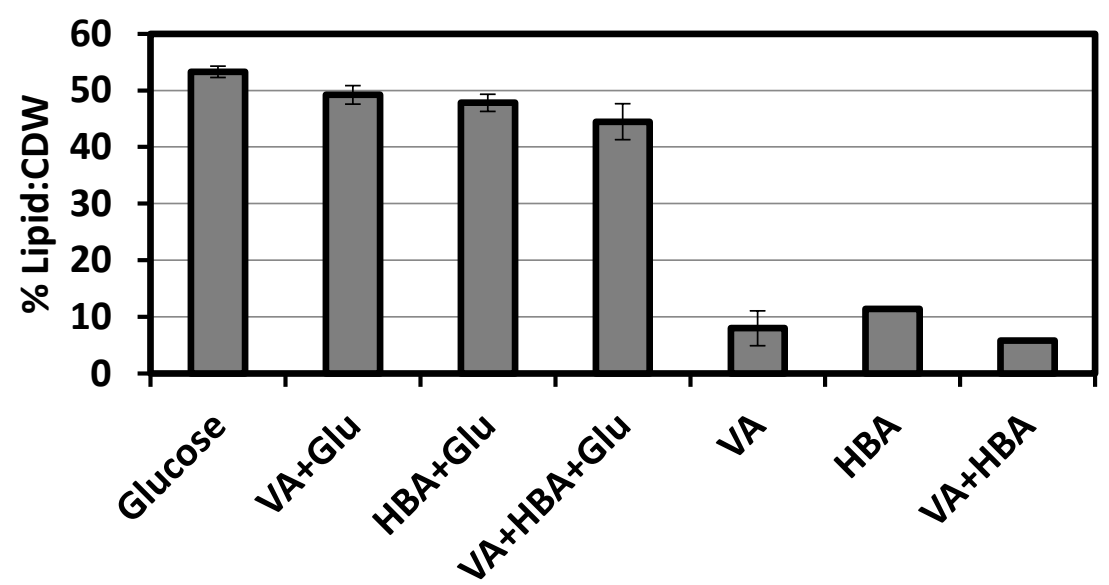

Figure 2. $R$. rhodochrous cell dry weight $(\mathbf{A} ; g / L \pm S D)$ and percent lipid accumulation $(\mathbf{B} ; \pm S D)$ of shake flask cultures supplemented with VA, HBA, VA + HBA, with and without glucose. Data shown from the 72-hour time point. 
A

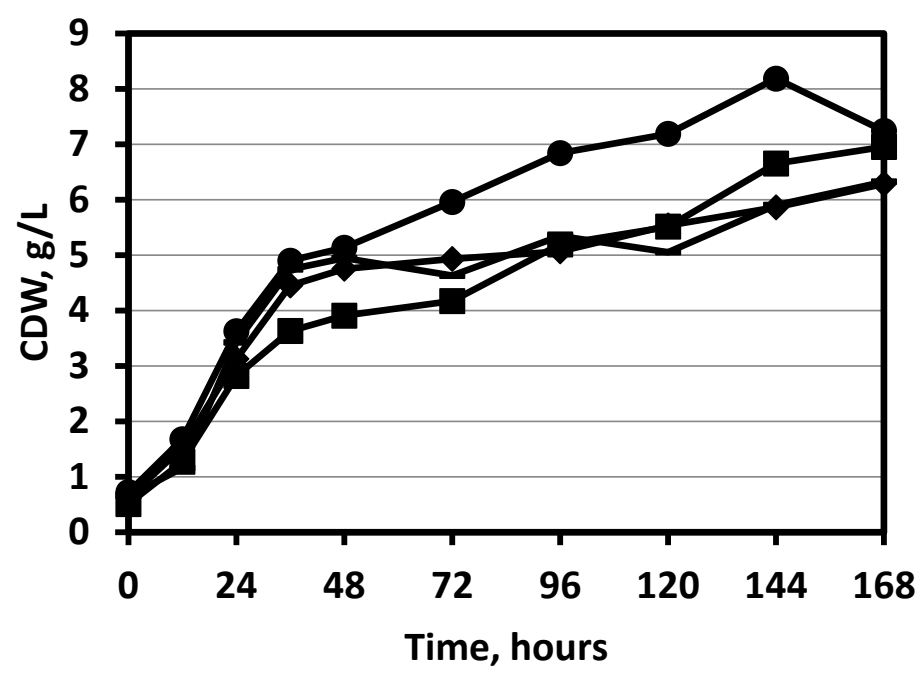

B

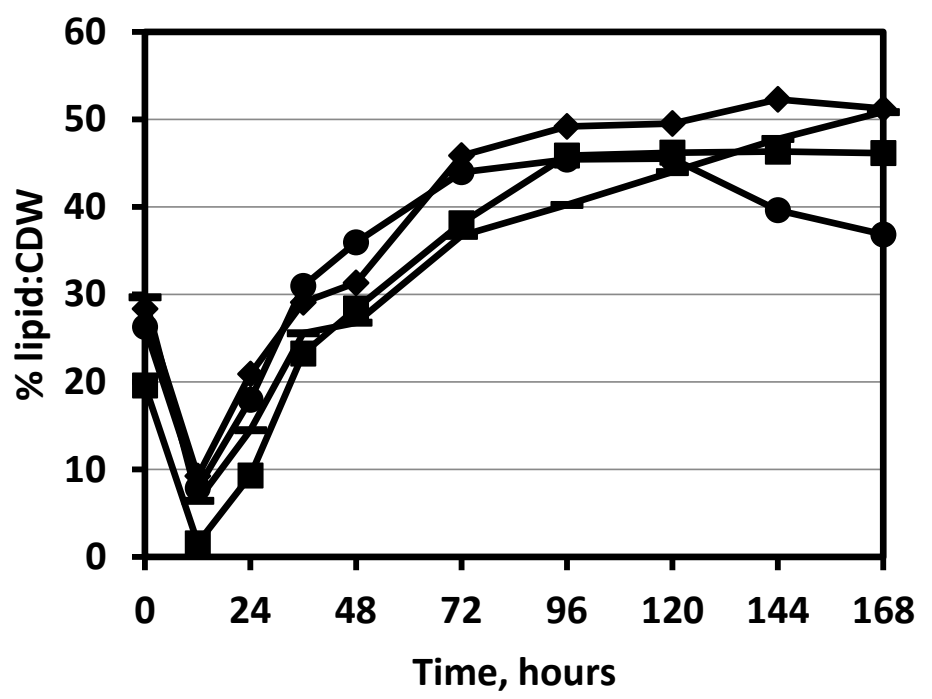

C

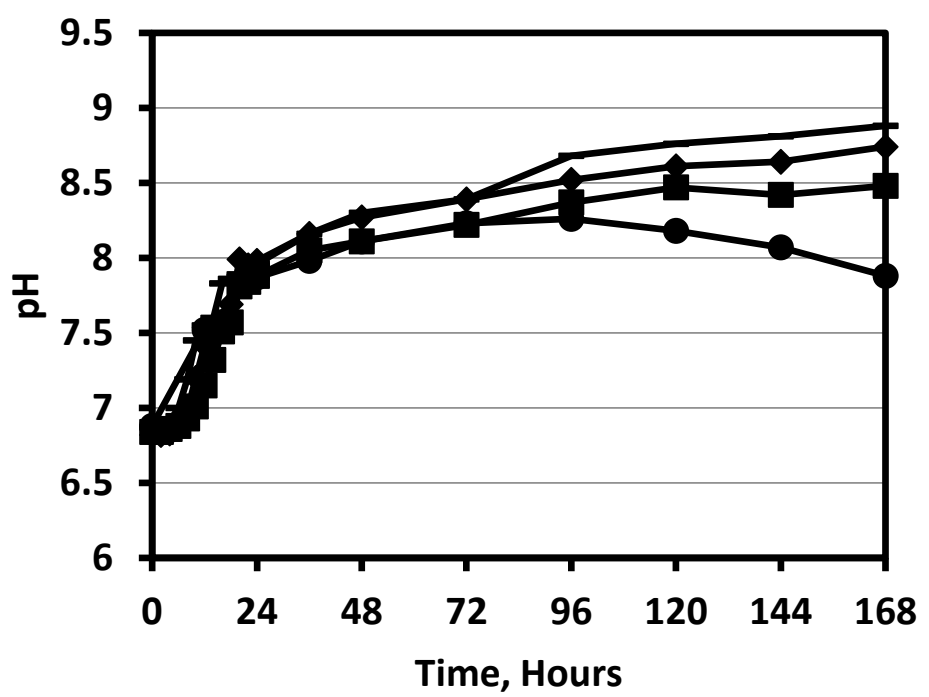


D

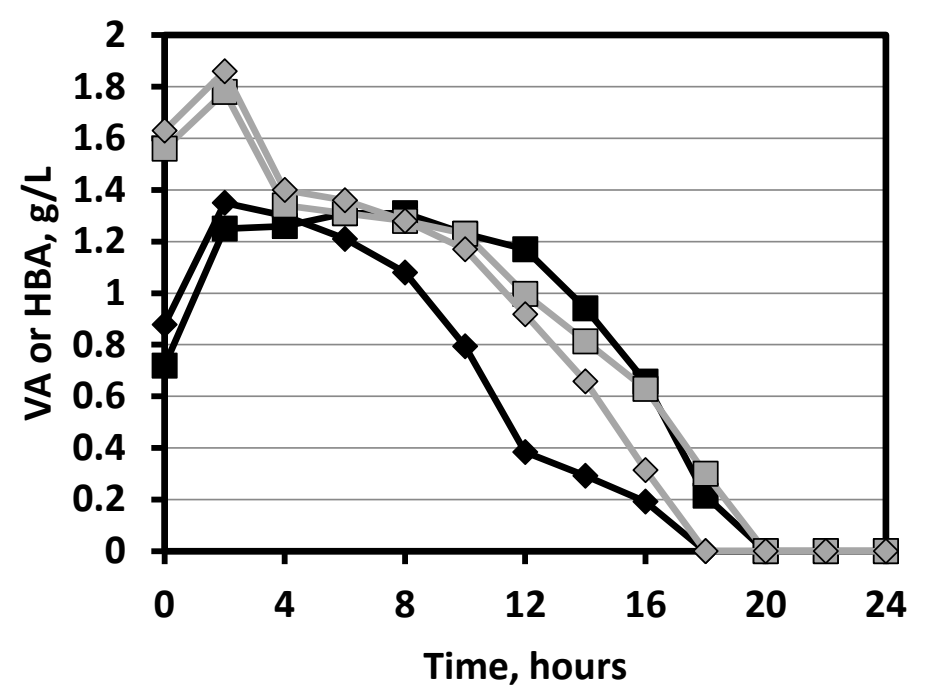

Figure 3. R. rhodochrous cell dry weight (A; g/L), percent lipid accumulation $(\mathbf{B})$ and $\mathrm{pH}$ (C) of 3L fermentations supplemented with glucose $(\bullet)$ and VA( $(\bullet), \mathrm{HBA}(\bullet)$, or VA + HBA (-). Vanillic acid and 4-hydroxybenzoic acid concentrations (D; g/L) were analyzed using HPLC-ELSD. Fermentation samples of VA (ם), HBA $(\bullet)$ and VA + HBA (grey; $\square, \bullet)$ were taken every 2 hours and filtered before analysis. 


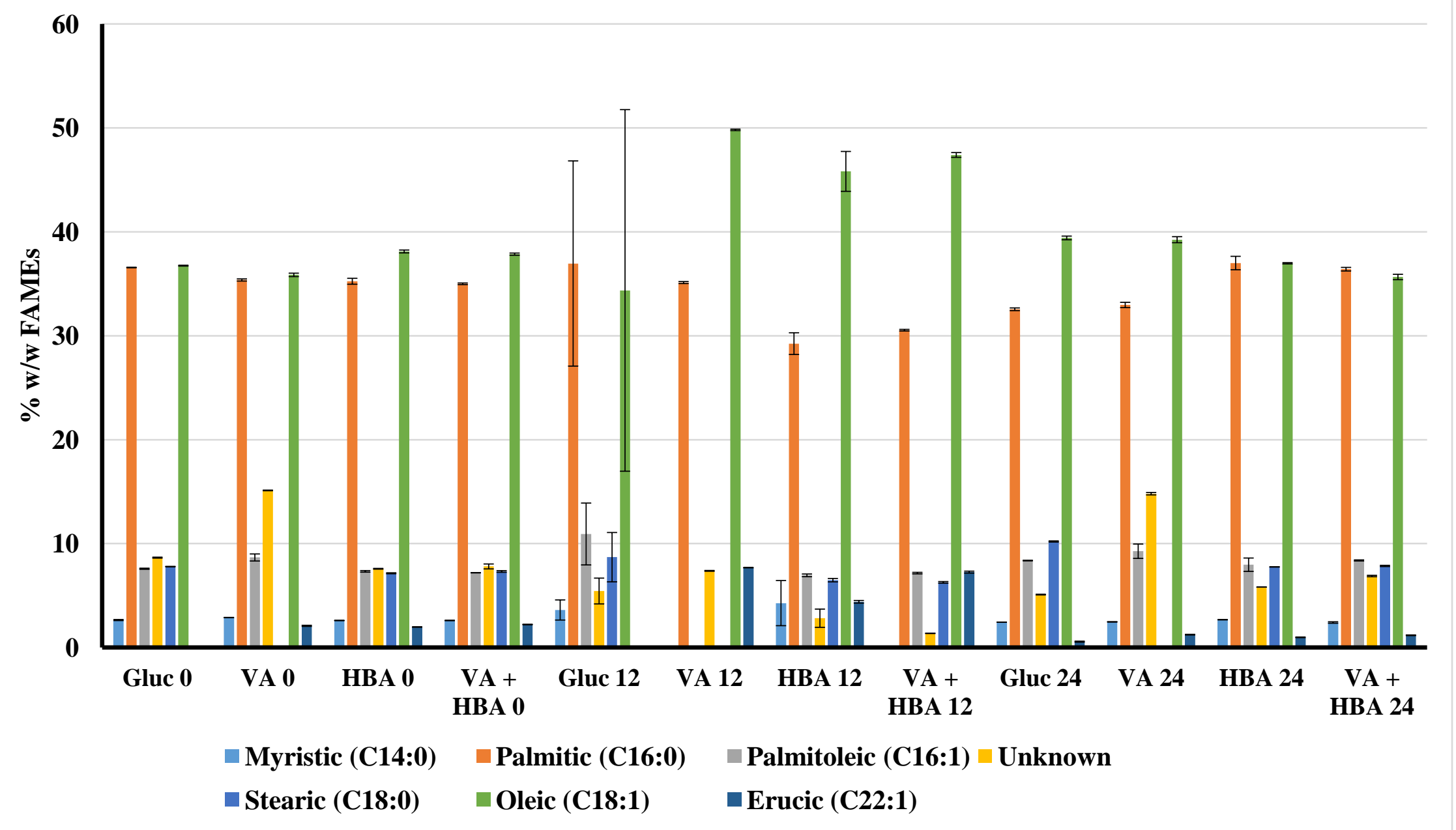

Figure 4. Fatty acid methyl ester profile (\% w/w) of $R$. rhodochrous grown in glucose (Glu), vanillic acid (VA), 4-hydroxybenzoic acid (HBA), or a mixture of VA and HBA (VA + HBA) at 0,12 , and 24 hours. Percentages are an average of triplicate injections of all experimental treatment replicates. Standard deviation is shown by error bars. FAMEs less than $1 \%$ are not shown. 
Table 1. Fatty acid methyl ester profile (\% w/w) of $R$. rhodochrous grown in glucose (Glu), phenol (Phe) or a mixture of glucose and phenol (GP). Percentages are an average of triplicate injections of all experimental treatment replicates. Standard deviation is less than $11 \%$. FAMEs less than $1 \%$ are not shown.

\begin{tabular}{llllllll}
\hline & $\begin{array}{l}\text { Myristic } \\
(\mathbf{C 1 4 : 0 )}\end{array}$ & $\begin{array}{l}\text { Palmitic } \\
(\mathbf{C 1 6 : 0 )}\end{array}$ & $\begin{array}{l}\text { Palmitoleic } \\
(\mathbf{C 1 6 : 1 )}\end{array}$ & $\begin{array}{l}\text { Unknown } \\
\text { (C18:0) }\end{array}$ & $\begin{array}{l}\text { Otearic } \\
\text { (C18:1) }\end{array}$ & $\begin{array}{l}\text { Erucic } \\
\text { (C22:1) }\end{array}$ \\
\hline \hline Glu 0H & 2.44 & 39.42 & 9.22 & 8.70 & 4.30 & 32.96 & 2.96 \\
Phe 0H & 2.48 & 39.61 & 9.48 & 8.61 & 4.37 & 32.51 & 2.95 \\
GP 0H & 2.53 & 39.32 & 9.69 & 8.61 & 4.20 & 32.77 & 2.88 \\
Glu 24H & 2.46 & 34.26 & 10.00 & 7.40 & 5.91 & 37.13 & 2.01 \\
Phe 24H & 2.75 & 36.18 & 9.12 & 8.02 & 4.72 & 36.53 & 2.37 \\
GP 24H & 3.26 & 38.60 & 13.22 & 7.28 & 4.12 & 29.88 & 3.60 \\
Glu 48H & 2.46 & 33.08 & 10.13 & 9.29 & 4.27 & 37.48 & 2.29 \\
Phe 48H & 2.90 & 37.31 & 10.10 & 7.41 & 3.73 & 32.86 & 4.98 \\
GP 48H & 3.15 & 38.73 & 8.73 & 13.48 & 4.05 & 28.41 & 2.38 \\
Glu 72H & 2.85 & 34.70 & 10.47 & 12.81 & 3.50 & 35.15 & 0.00 \\
Phe 72H & 0.92 & 49.89 & 7.62 & 4.04 & 1.42 & 36.10 & 0.00 \\
GP 72H & 3.45 & 37.14 & 15.05 & 14.19 & 3.86 & 25.83 & 0.00 \\
\hline
\end{tabular}


Table 2. Proteins of $R$. rhodochrous cultured in glucose, phenol, and glucose with phenol.

\begin{tabular}{|c|c|c|c|c|c|}
\hline $\begin{array}{l}\text { Glucose } 24 \text { hrs } \\
\text { vs Glucose } 0 \text { hrs } \\
\text { Fold change }\end{array}$ & $\begin{array}{l}\text { Fisher's } \\
\text { Exact Tes } \\
\text { (P-Value): } \\
*(\mathbf{p}<= \\
\mathbf{0 . 0 0 6 1 0}) \\
\end{array}$ & Protein Name & Role & $\begin{array}{l}\text { Accession } \\
\text { Number }\end{array}$ & $\begin{array}{c}\text { Molecular } \\
\text { Weight }\end{array}$ \\
\hline-50.0 & $<0.00010$ & Adenylate kinase [Rhodococcus rhodochrous ATCC 21198] & energy balance & gi|573523828 & $19 \mathrm{kDa}$ \\
\hline-33.3 & $<0.00010$ & $\begin{array}{l}\text { ABC-type branched-chain amino acid transport systems periplasmic component-like protein } \\
\text { [Rhodococcus rhodochrous ATCC 21198] }\end{array}$ & transporter & gi|573527736 & $45 \mathrm{kDa}$ \\
\hline-16.7 & $<0.00010$ & malic protein NAD-binding protein [Rhodococcus rhodochrous ATCC 21198] & fatty acid synthesis & gi|573526831 & $41 \mathrm{kDa}$ \\
\hline-14.3 & $<0.00010$ & Succinyl-CoA ligase (ADP-forming) subunit beta [Rhodococcus rhodochrous ATCC 21198] & central metabolism & gi|573528361 & $41 \mathrm{kDa}$ \\
\hline-10.0 & 0.0023 & transketolase [Rhodococcus rhodochrous ATCC 21198] & central metabolism & gi|573525360 & $74 \mathrm{kDa}$ \\
\hline-5.0 & $<0.00010$ & 6-phosphogluconate dehydratase [Rhodococcus rhodochrous ATCC 21198] & central metabolism & gi|573526218 & $64 \mathrm{kDa}$ \\
\hline-3.3 & $<0.00010$ & Enolase [Rhodococcus rhodochrous ATCC 21198] & central metabolism & gi|573525840 & $45 \mathrm{kDa}$ \\
\hline-3.3 & $<0.00010$ & Phosphoglycerate kinase [Rhodococcus rhodochrous ATCC 21198] & central metabolism & gi|573525373 & $42 \mathrm{kDa}$ \\
\hline-2.0 & 0.005 & $\begin{array}{l}\text { 2-oxoglutarate dehydrogenase, E2 component, dihydrolipoamide succinyltransferase } \\
\text { [Rhodococcus rhodochrous ATCC 21198] }\end{array}$ & central metabolism & gi|573526276 & $61 \mathrm{kDa}$ \\
\hline-2.0 & 0.0027 & nitrogen regulatory protein P-II [Rhodococcus rhodochrous ATCC 21198] & nitrogen metabolism & gi|573526092 & $12 \mathrm{kDa}$ \\
\hline-2.0 & $<0.00010$ & glyceraldehyde-3-phosphate dehydrogenase, type I [Rhodococcus rhodochrous ATCC 21198] & central metabolism & gi|573525374 & $36 \mathrm{kDa}$ \\
\hline 2.5 & $<0.00010$ & dihydrolipoamide dehydrogenase [Rhodococcus rhodochrous ATCC 21198] & central metabolism & gi|573523617 & $57 \mathrm{kDa}$ \\
\hline 3.2 & 0.0028 & Citrate (Si)-synthase [Rhodococcus rhodochrous ATCC 21198] & central metabolism & gi|573528494 & $40 \mathrm{kDa}$ \\
\hline 4.3 & $<0.00010$ & pyruvate carboxylase [Rhodococcus rhodochrous ATCC 21198] & central metabolism & gi|573529586 & $121 \mathrm{kDa}$ \\
\hline 5.2 & $<0.00010$ & Acyl transferase [Rhodococcus rhodochrous ATCC 21198] & fatty acid synthesis & gi|573527113 & $323 \mathrm{kDa}$ \\
\hline 5.3 & $<0.00010$ & Biotin carboxylase, Propionyl-CoA carboxylase [Rhodococcus rhodochrous ATCC 21198] & fatty acid synthesis & gi|573528207 & $199 \mathrm{kDa}$ \\
\hline 5.5 & 0.00047 & 3-isopropylmalate dehydrogenase [Rhodococcus rhodochrous ATCC 21198] & central metabolism & gi|573529634 & $35 \mathrm{kDa}$ \\
\hline 15.0 & 0.00056 & isocitrate lyase [Rhodococcus rhodochrous ATCC 21198] & central metabolism & gi|573523598 & $47 \mathrm{kDa}$ \\
\hline
\end{tabular}




\begin{tabular}{|c|c|c|c|c|c|}
\hline $\begin{array}{c}\text { Glucose } 24 \text { hrs } \\
\text { vs } \\
\text { Glucose+phenol } \\
24 \text { hrs } \\
\text { Fold Change }\end{array}$ & $\begin{array}{c}\text { Fisher's } \\
\text { Exact Tes } \\
\text { (P-Value) } \\
*(\mathbf{p}<= \\
\mathbf{0 . 0 1 1 2 8})\end{array}$ & Protein Name & Role & $\begin{array}{l}\text { Accession } \\
\text { Number }\end{array}$ & $\begin{array}{l}\text { Molecular } \\
\text { Weight }\end{array}$ \\
\hline-33.3 & $<0.00010$ & Succinyl-CoA ligase (ADP-forming) subunit beta [Rhodococcus rhodochrous ATCC 21198] & central metabolism & gi|573528361 & $41 \mathrm{kDa}$ \\
\hline-25.0 & $<0.00010$ & malic protein NAD-binding protein [Rhodococcus rhodochrous ATCC 21198] & fatty acid metabolism & gi|573526831 & $41 \mathrm{kDa}$ \\
\hline-12.5 & $<0.00010$ & catechol 1,2-dioxygenase [Rhodococcus rhodochrous ATCC 21198] & phenol degradation & gi|573524225 & $32 \mathrm{kDa}$ \\
\hline-11.1 & $<0.00010$ & glutamate synthase, NADH/NADPH, small subunit [Rhodococcus rhodochrous ATCC 21198] & central metabolism & gi|573526911 & $52 \mathrm{kDa}$ \\
\hline-10.0 & 0.0016 & glucokinase, ROK family [Rhodococcus rhodochrous ATCC 21198] & central metabolism & gi|573526222 & $33 \mathrm{kDa}$ \\
\hline-10.0 & 0.0022 & $\begin{array}{l}\text { 2,3-bisphosphoglycerate-dependent phosphoglycerate mutase [Rhodococcus rhodochrous ATCC } \\
\text { 21198] }\end{array}$ & central metabolism & gi|573523521 & $27 \mathrm{kDa}$ \\
\hline-10.0 & 0.00012 & UTP--glucose-1-phosphate uridylyltransferase [Rhodococcus rhodochrous ATCC 21198] & nitrogen metabolism & gi|573528320 & $32 \mathrm{kDa}$ \\
\hline-5.0 & 0.0077 & transketolase [Rhodococcus rhodochrous ATCC 21198] & central metabolism & gi|573525360 & $74 \mathrm{kDa}$ \\
\hline-3.3 & 0.007 & Transaldolase [Rhodococcus rhodochrous ATCC 21198] & central metabolism & gi|573525361 & $41 \mathrm{kDa}$ \\
\hline-2.0 & $<0.00010$ & Phosphoglycerate kinase [Rhodococcus rhodochrous ATCC 21198] & central metabolism & gi|573525373 & $42 \mathrm{kDa}$ \\
\hline-1.7 & $<0.00010$ & Enolase [Rhodococcus rhodochrous ATCC 21198] & central metabolism & gi|573525840 & $45 \mathrm{kDa}$ \\
\hline 1.6 & 0.0043 & pyruvate carboxylase [Rhodococcus rhodochrous ATCC 21198] & Anaplerotic pathway & gi|573529586 & $121 \mathrm{kDa}$ \\
\hline 1.7 & 0.00034 & aconitate hydratase 1 [Rhodococcus rhodochrous ATCC 21198] & central metabolism & gi|573527327 & $101 \mathrm{kDa}$ \\
\hline 2.1 & 0.0018 & 3-isopropylmalate dehydrogenase [Rhodococcus rhodochrous ATCC 21198] & citrate/malate cyle & gi|573529634 & $35 \mathrm{kDa}$ \\
\hline 2.3 & $<0.00010$ & fructose-1,6-bisphosphatase, class II [Rhodococcus rhodochrous ATCC 21198] & central metabolism & gi|573526483 & $36 \mathrm{kDa}$ \\
\hline 2.3 & $<0.00010$ & Triosephosphate isomerase, bacterial/eukaryotic [Rhodococcus rhodochrous ATCC 21198] & central metabolism & gi|573525372 & $27 \mathrm{kDa}$ \\
\hline 2.3 & 0.00074 & Biotin carboxylase, Oxaloacetate decarboxylase [Rhodococcus rhodochrous ATCC 21198] & fatty acid metabolism/ ACC & gi|573525143 & $64 \mathrm{kDa}$ \\
\hline 2.5 & $<0.00010$ & dihydrolipoamide dehydrogenase [Rhodococcus rhodochrous ATCC 21198] & central metabolism & gi|573523617 & $57 \mathrm{kDa}$ \\
\hline 2.6 & 0.0046 & 6-phosphogluconate dehydrogenase, decarboxylating [Rhodococcus rhodochrous ATCC 21198] & central metabolism & gi|573524711 & $52 \mathrm{kDa}$ \\
\hline 2.9 & $<0.00010$ & cysteine synthase A [Rhodococcus rhodochrous ATCC 21198] & Amino acid and protein metab & gi|573523949 & $33 \mathrm{kDa}$ \\
\hline
\end{tabular}


2-oxo-acid dehydrogenase E1 subunit, homodimeric type [Rhodococcus rhodochrous ATCC

5.6

5.7

7.2

7.9

$$
8.2
$$

\section{Glucose 24 hrs}

vs

\section{Phenol 24 hrs}

$<0.00010 \quad 21198$

$<0.00010$ Acyl transferase [Rhodococcus rhodochrous ATCC 21198]

$<0.00010$ urea ABC transporter, urea binding protein [Rhodococcus rhodochrous ATCC 21198]

0.00046 Acetyl-coenzyme A synthetase [Rhodococcus rhodochrous ATCC 21198]

0.00048 IMP dehydrogenase family protein [Rhodococcus rhodochrous ATCC 21198]

$<0.00010$ Biotin carboxylase, Propionyl-CoA carboxylase [Rhodococcus rhodochrous ATCC 21198]

$<0.00010$ succinate dehydrogenase, flavoprotein subunit [Rhodococcus rhodochrous ATCC 21198]

succinate dehydrogenase and fumarate reductase iron-sulfur protein [Rhodococcus rhodochrous

$<0.00010 \quad$ ATCC 21198]

0.00089 3-oxoacyl-(acyl-carrier-protein) reductase [Rhodococcus rhodochrous ATCC 21198]

$<0.00010$ Beta-ketoacyl-acyl-carrier-protein synthase III [Rhodococcus rhodochrous ATCC 21198]

$<0.00010$ Catalase-peroxidase [Rhodococcus rhodochrous ATCC 21198]

$<0.00010$ acetyl-CoA acetyltransferase [Rhodococcus rhodochrous ATCC 21198]

Fisher's

Exact Tes

(P-Value)

Protein Name

$0.01037)$

$<0.00010$ malic protein NAD-binding protein [Rhodococcus rhodochrous ATCC 21198]

$-50.0$

$-33.3$

$<0.00010$ putative HpaB/PvcC/4-BUDH, partial [Rhodococcus rhodochrous ATCC 21198]

$<0.00010$ Catechol 2,3-dioxygenase [Rhodococcus rhodochrous ATCC 21198]

$-33.3$

$<0.00010$ Succinyl-CoA ligase (ADP-forming) subunit beta [Rhodococcus rhodochrous ATCC 21198]

$<0.00010$ catechol 1,2-dioxygenase [Rhodococcus rhodochrous ATCC 21198]

$<0.00010$ Adenylate kinase [Rhodococcus rhodochrous ATCC 21198]

$<0.00010$ putative $\mathrm{HpaB} / \mathrm{PvcC} / 4-\mathrm{BUDH}$, partial [Rhodococcus rhodochrous ATCC 21198]

$<0.00010$ catechol 1,2-dioxygenase [Rhodococcus rhodochrous ATCC 21198]

$<0.00010$ Acetaldehyde dehydrogenase [Rhodococcus rhodochrous ATCC 21198]

$-12.5$

$-11.1$

$-11.1$

$<0.00010$ 2,3-bisphosphoglycerate-dependent phosphoglycerate mutase [Rhodococcus rhodochrous ATCC 21198]

$<0.00010$ putative HpaB/PvcC/4-BUDH, partial [Rhodococcus rhodochrous ATCC 21198]

0.00033 glutamate synthase, NADH/NADPH, small subunit [Rhodococcus rhodochrous ATCC 21198]

central metabolism

fatty acid metabolism

Nitrogen metabolism

fatty acid metabolism

citrate/malate cycle

central metabolism

Amino acid and protein metabolism

Amino acid and protein metabolism

fatty acid metabolism

fatty acid metabolism

antioxidant

B-oxidation gi|573525512 $105 \mathrm{kDa}$

gi|573527113 $323 \mathrm{kDa}$

gi|573523602 $44 \mathrm{kDa}$

gi $573529490 \quad 70 \mathrm{kDa}$

gi| $573524713 \quad 50 \mathrm{kDa}$

gi|573528207 $199 \mathrm{kDa}$

gi|573525085 $64 \mathrm{kDa}$

gi|573525084 $29 \mathrm{kDa}$

gi $573527700 \quad 46 \mathrm{kDa}$

gi|573528205 $71 \mathrm{kDa}$

gi|573526986 $80 \mathrm{kDa}$

gi|573527699 $46 \mathrm{kDa}$

\begin{tabular}{lll}
\multicolumn{1}{c}{ Role } & $\begin{array}{c}\text { Accession } \\
\text { Number }\end{array}$ & $\begin{array}{c}\text { Molecular } \\
\text { Weight }\end{array}$ \\
\hline fatty acid metabolism & gi|573526831 & $41 \mathrm{kDa}$ \\
phenol degradation & gi|573528977 & $36 \mathrm{kDa}$ \\
phenol metabolism & gi|573526750 & $41 \mathrm{kDa}$ \\
gi|573528361 & $41 \mathrm{kDa}$ \\
Central metabolism & & \\
phenol metabolism & gi|573523683 & $31 \mathrm{kDa}$ \\
energy balance & gi|573523828 & $19 \mathrm{kDa}$ \\
phenol degradation & gi|573524229 & $11 \mathrm{kDa}$ \\
phenol metabolism & gi 573524225 & $32 \mathrm{kDa}$ \\
central metabolism & gi|573526749 & $31 \mathrm{kDa}$ \\
central metabolism & gi|573523521 & $27 \mathrm{kDa}$ \\
& & \\
phenol degradation & gi 573525281 & $17 \mathrm{kDa}$ \\
central metabolism & gi|573526911 & $52 \mathrm{kDa}$
\end{tabular}




\begin{tabular}{|c|c|c|c|c|c|}
\hline-10.0 & 0.0072 & PEP phosphonomutase-like protein [Rhodococcus rhodochrous ATCC 21198] & central metabolism & gi|573524216 & $26 \mathrm{kDa}$ \\
\hline-10.0 & 0.0016 & Alcohol dehydrogenase, zinc-type, actinomycete [Rhodococcus rhodochrous ATCC 21198] & energy balance & gi|573527429 & $39 \mathrm{kDa}$ \\
\hline-10.0 & 0.01 & Phosphoenolpyruvate carboxykinase (GTP) [Rhodococcus rhodochrous ATCC 21198] & fatty acid metabolism & gi|573523953 & $67 \mathrm{kDa}$ \\
\hline-10.0 & 0.0016 & acetyl-CoA acetyltransferase [Rhodococcus rhodochrous ATCC 21198] & B oxidation & gi|573524197 & $42 \mathrm{kDa}$ \\
\hline-2.0 & $<0.00010$ & Phosphoglycerate kinase [Rhodococcus rhodochrous ATCC 21198] & central metabolism & gi|573525373 & $42 \mathrm{kDa}$ \\
\hline-1.7 & 0.002 & Enolase [Rhodococcus rhodochrous ATCC 21198] & central metabolism & gi|573525840 & $45 \mathrm{kDa}$ \\
\hline 1.5 & 0.0075 & aconitate hydratase 1 [Rhodococcus rhodochrous ATCC 21198] & central metabolism & gi|573527327 & $101 \mathrm{kDa}$ \\
\hline 1.6 & 0.0037 & Triosephosphate isomerase, bacterial/eukaryotic [Rhodococcus rhodochrous ATCC 21198] & central metabolism & gi|573525372 & $27 \mathrm{kDa}$ \\
\hline 1.6 & $0.003^{\circ}$ & Triosephosphate isomerase, bacterial/eukaryotic [Rhodococcus rhodochrous ATCC 21198] & central metabolism & gi|573525372 & $27 \mathrm{kDa}$ \\
\hline 2.3 & 0.0015 & 3-isopropylmalate dehydrogenase [Rhodococcus rhodochrous ATCC 21198] & malate cycle & gi|573529634 & $35 \mathrm{kDa}$ \\
\hline 2.5 & 0.00092 & Biotin carboxylase, Oxaloacetate decarboxylase [Rhodococcus rhodochrous ATCC 21198] & fatty acid metabolism & gi|573525143 & $64 \mathrm{kDa}$ \\
\hline 2.7 & 0.0027 & succinate dehydrogenase, flavoprotein subunit [Rhodococcus rhodochrous ATCC 21198] & $\mathrm{n}$ & gi|573525085 & $64 \mathrm{kDa}$ \\
\hline 2.8 & 0.0053 & 6-phosphogluconate dehydrogenase, decarboxylating [Rhodococcus rhodochrous ATCC 21198] & central metabolism & gi|573524711 & $52 \mathrm{kDa}$ \\
\hline 2.8 & $<0.00010$ & D-3-phosphoglycerate dehydrogenase [Rhodococcus rhodochrous ATCC 21198] & fatty acid metabolism & gi|573529635 & $55 \mathrm{kDa}$ \\
\hline 2.9 & $<0.00010$ & fructose-1,6-bisphosphatase, class II [Rhodococcus rhodochrous ATCC 21198] & central metabolism & gi|573526483 & $36 \mathrm{kDa}$ \\
\hline 3.1 & $<0.00010$ & dihydrolipoamide dehydrogenase [Rhodococcus rhodochrous ATCC 21198] & Acetyl-CoA synthesis & gi|573523617 & $57 \mathrm{kDa}$ \\
\hline 3.1 & $<0.00010$ & pyruvate carboxylase [Rhodococcus rhodochrous ATCC 21198] & central metabolism & gi|573529586 & $121 \mathrm{kDa}$ \\
\hline 3.3 & 0.00065 & IMP dehydrogenase family protein [Rhodococcus rhodochrous ATCC 21198] & citrate/malate cycle & gi|573524713 & $50 \mathrm{kDa}$ \\
\hline 4.2 & 0.00076 & Glucose-6-phosphate isomerase [Rhodococcus rhodochrous ATCC 21198] & central metabolism & gi|573528367 & $58 \mathrm{kDa}$ \\
\hline 4.5 & 0.0042 & IMP dehydrogenase [Rhodococcus rhodochrous ATCC 21198] & citrate/malate & gi|573524120 & $52 \mathrm{kDa}$ \\
\hline 4.5 & 0.0027 & acetyl-CoA acetyltransferase [Rhodococcus rhodochrous ATCC 21198] & B oxidation & gi|573527699 & $46 \mathrm{kDa}$ \\
\hline 5.5 & $<0.00010$ & Phosphoserine aminotransferase [Rhodococcus rhodochrous ATCC 21198] & fatty acid metabolism & gi|573528495 & $40 \mathrm{kDa}$ \\
\hline 7.4 & 0.00063 & 6-phosphogluconate dehydratase [Rhodococcus rhodochrous ATCC 21198] & central metabolism & gi|573526218 & $64 \mathrm{kDa}$ \\
\hline 9.0 & $<0.00010$ & Beta-ketoacyl-acyl-carrier-protein synthase III [Rhodococcus rhodochrous ATCC 21198] & fatty acid metabolism & gi|573528205 & $71 \mathrm{kDa}$ \\
\hline 44.0 & $<0.00010$ & Acyl transferase [Rhodococcus rhodochrous ATCC 21198] & fatty acid metabolism & gi|573527113 & $323 \mathrm{kDa}$ \\
\hline 78.0 & $<0.00010$ & Biotin carboxylase, Propionyl-CoA carboxylase [Rhodococcus rhodochrous ATCC 21198] & fatty acid metabolism & gi|573528207 & $199 \mathrm{kDa}$ \\
\hline $\begin{array}{l}\text { Phenol } 24 \text { hrs } \\
\text { vs Phenol } 0 \text { hrs } \\
\text { Fold Change }\end{array}$ & $\begin{array}{c}\text { Fisher's } \\
\text { Exact Tes } \\
\text { (P-Value) } \\
*(p<=\end{array}$ & Protein Name & Role & $\begin{array}{c}\text { Accession } \\
\text { Number }\end{array}$ & $\begin{array}{c}\text { Molecular } \\
\text { Weight }\end{array}$ \\
\hline
\end{tabular}


0.00579)

\begin{tabular}{|c|c|c|c|c|c|}
\hline-33.3 & $<0.00010$ & 6-phosphogluconate dehydratase [Rhodococcus rhodochrous ATCC 21198] & central metabolism & gi|573526218 & $64 \mathrm{kDa}$ \\
\hline-20.0 & $<0.00010$ & $\begin{array}{l}\text { ABC-type branched-chain amino acid transport systems periplasmic component-like protein } \\
\text { [Rhodococcus rhodochrous ATCC 21198] }\end{array}$ & transporter & gi|573527736 & $45 \mathrm{kDa}$ \\
\hline-14.3 & $<0.00010$ & Biotin carboxylase, Propionyl-CoA carboxylase [Rhodococcus rhodochrous ATCC 21198] & fatty acid metabolism & gi|573528207 & $199 \mathrm{kDa}$ \\
\hline-10.0 & $<0.00010$ & Acyl transferase [Rhodococcus rhodochrous ATCC 21198] & fatty acid metabolism & gi|573527113 & $323 \mathrm{kDa}$ \\
\hline-2.5 & 0.00018 & aspartate-semialdehyde dehydrogenase [Rhodococcus rhodochrous ATCC 21198] & amino acid metabolism & gi|573524856 & $36 \mathrm{kDa}$ \\
\hline-2.5 & 0.0043 & urea $\mathrm{ABC}$ transporter, urea binding protein [Rhodococcus rhodochrous ATCC 21198] & nitrogen metabolism & gi|573523602 & $44 \mathrm{kDa}$ \\
\hline-2.5 & $<0.00010$ & glyceraldehyde-3-phosphate dehydrogenase, type I [Rhodococcus rhodochrous ATCC 21198] & Central metabolism & gi|573525374 & $36 \mathrm{kDa}$ \\
\hline-2.0 & 0.0015 & $\begin{array}{l}\text { 2-oxoglutarate dehydrogenase, E2 component, dihydrolipoamide succinyltransferase } \\
\text { [Rhodococcus rhodochrous ATCC 21198] }\end{array}$ & central metabolism & gi|573526276 & $61 \mathrm{kDa}$ \\
\hline-2.0 & 0.0013 & D-3-phosphoglycerate dehydrogenase [Rhodococcus rhodochrous ATCC 21198] & amino acid metabolism & gi|573529635 & $55 \mathrm{kDa}$ \\
\hline-2.0 & 0.00036 & Adenylate kinase [Rhodococcus rhodochrous ATCC 21198] & energy balance & gi|573523828 & $19 \mathrm{kDa}$ \\
\hline-2.0 & $<0.00010$ & Enolase [Rhodococcus rhodochrous ATCC 21198] & central metabolism & gi| 573525840 & $45 \mathrm{kDa}$ \\
\hline-1.4 & 0.0035 & Phosphoglycerate kinase [Rhodococcus rhodochrous ATCC 21198] & central metabolism & gi $\mid 573525373$ & $42 \mathrm{kDa}$ \\
\hline 2.7 & 0.00013 & malic protein NAD-binding protein [Rhodococcus rhodochrous ATCC 21198] & fatty acid metabolism & gi|573526831 & $41 \mathrm{kDa}$ \\
\hline 9.9 & 0.0046 & Biotin carboxylase, Oxaloacetate decarboxylase [Rhodococcus rhodochrous ATCC 21198] & fatty acid metabolism/ACC & gi|573525143 & $64 \mathrm{kDa}$ \\
\hline 11.0 & 0.0027 & $\begin{array}{l}\text { 2,3-bisphosphoglycerate-dependent phosphoglycerate mutase [Rhodococcus rhodochrous ATCC } \\
\text { 21198] }\end{array}$ & fatty acid metabolism & gi| 573523521 & $27 \mathrm{kDa}$ \\
\hline 11.0 & 0.0027 & putative $\mathrm{HpaB} / \mathrm{PvcC} / 4-\mathrm{BUDH}$, partial [Rhodococcus rhodochrous ATCC 21198] & phenol degradation & gi|573525281 & $17 \mathrm{kDa}$ \\
\hline 12.0 & 0.00093 & Transaldolase [Rhodococcus rhodochrous ATCC 21198] & central metabolism & gi|573525361 & $41 \mathrm{kDa}$ \\
\hline 13.0 & 0.0016 & Acetaldehyde dehydrogenase [Rhodococcus rhodochrous ATCC 21198] & central metabolism & gi|573526749 & $31 \mathrm{kDa}$ \\
\hline 15.0 & 0.00054 & isocitrate lyase [Rhodococcus rhodochrous ATCC 21198] & Dioxylate/dicarboxylate metabolism & gi| 573523598 & $47 \mathrm{kDa}$ \\
\hline 16.0 & $<0.00010$ & Betaine-aldehyde dehydrogenase [Rhodococcus rhodochrous ATCC 21198] & central metabolism & gi| 573526747 & $52 \mathrm{kDa}$ \\
\hline 19.0 & $<0.00010$ & putative $\mathrm{HpaB} / \mathrm{PvcC} / 4-\mathrm{BUDH}$, partial [Rhodococcus rhodochrous ATCC 21198] & phenol degradation & gi|573524229 & $11 \mathrm{kDa}$ \\
\hline 20.0 & $<0.00010$ & catechol 1,2-dioxygenase [Rhodococcus rhodochrous ATCC 21198] & phenol degradation & gi $\mid 573524225$ & $32 \mathrm{kDa}$ \\
\hline 23.0 & $<0.00010$ & catechol 1,2-dioxygenase [Rhodococcus rhodochrous ATCC 21198] & phenol degradation & gi| 573523683 & $31 \mathrm{kDa}$ \\
\hline 31.0 & $<0.00010$ & Catechol 2,3-dioxygenase [Rhodococcus rhodochrous ATCC 21198] & phenol degradation & gi $\mid 573526750$ & $41 \mathrm{kDa}$ \\
\hline 54.0 & $<0.00010$ & putative $\mathrm{HpaB} / \mathrm{PvcC} / 4-\mathrm{BUDH}$, partial [Rhodococcus rhodochrous ATCC 21198] & phenol degradation & gi|573528977 & $36 \mathrm{kDa}$ \\
\hline
\end{tabular}




\begin{tabular}{|c|c|c|c|c|c|}
\hline $\begin{array}{l}\text { Phenol+glucose } \\
24 \text { hrs vs } 0 \text { hrs } \\
\text { Fold Change }\end{array}$ & $\begin{array}{c}\text { Fisher's } \\
\text { Exact Tes } \\
\text { (P-Value) } \\
*(\mathbf{p}<= \\
\mathbf{0 . 0 0 6 1 0})\end{array}$ & Protein Name & Role & $\begin{array}{l}\text { Accession } \\
\text { Number }\end{array}$ & $\begin{array}{l}\text { Molecular } \\
\text { Weight }\end{array}$ \\
\hline-33.3 & $<0.00010$ & $\begin{array}{l}\text { ABC-type branched-chain amino acid transport systems periplasmic component-like protein } \\
\text { [Rhodococcus rhodochrous ATCC } 21198 \text { ] }\end{array}$ & Transporter & gi|573527736 & $45 \mathrm{kDa}$ \\
\hline-10.0 & $<0.00010$ & ABC-type transporter, periplasmic subunit family 3 [Rhodococcus rhodochrous ATCC 21198] & transporter & gi|573527959 & $29 \mathrm{kDa}$ \\
\hline-5.0 & $<0.00010$ & Adenylate kinase [Rhodococcus rhodochrous ATCC 21198] & energy balance & gi|573523828 & $19 \mathrm{kDa}$ \\
\hline-5.0 & $<0.00010$ & urea ABC transporter, urea binding protein [Rhodococcus rhodochrous ATCC 21198] & Nitrogen metabolism & gi|573523602 & $44 \mathrm{kDa}$ \\
\hline-3.3 & $<0.00010$ & 6-phosphogluconate dehydratase [Rhodococcus rhodochrous ATCC 21198] & central metabolism & gi|573526218 & $64 \mathrm{kDa}$ \\
\hline-2.5 & $<0.00010$ & glyceraldehyde-3-phosphate dehydrogenase, type I [Rhodococcus rhodochrous ATCC 21198] & central metabolism & gi|573525374 & $36 \mathrm{kDa}$ \\
\hline-2.0 & $<0.00010$ & glutamine synthetase, type I [Rhodococcus rhodochrous ATCC 21198] & nitrogen metabolism & gi|573526282 & $54 \mathrm{kDa}$ \\
\hline-2.0 & 0.0011 & $\begin{array}{l}\text { 2-oxoglutarate dehydrogenase, E2 component, dihydrolipoamide succinyltransferase } \\
\text { [Rhodococcus rhodochrous ATCC 21198] }\end{array}$ & central metabolism & gi|573526276 & $61 \mathrm{kDa}$ \\
\hline-1.7 & $<0.00010$ & Enolase [Rhodococcus rhodochrous ATCC 21198] & central metabolism & gi|573525840 & $45 \mathrm{kDa}$ \\
\hline 2.1 & 0.0026 & Succinyl-CoA ligase (ADP-forming) subunit beta [Rhodococcus rhodochrous ATCC 21198] & central metabolism & gi|573528361 & $41 \mathrm{kDa}$ \\
\hline 4.9 & 0.0028 & 2-oxoglutarate dehydrogenase, E1 subunit [Rhodococcus rhodochrous ATCC 21198] & central metabolism & gi|573526837 & $138 \mathrm{kDa}$ \\
\hline 10.0 & 0.0045 & UTP--glucose-1-phosphate uridylyltransferase [Rhodococcus rhodochrous ATCC 21198] & Nitrogen metabolism & gi|573528320 & $32 \mathrm{kDa}$ \\
\hline 11.0 & 0.003 & Biotin carboxylase, Oxaloacetate decarboxylase [Rhodococcus rhodochrous ATCC 21198] & fatty acid metabolism/acc & gi|573525143 & $64 \mathrm{kDa}$ \\
\hline 11.0 & 0.003 & isocitrate lyase [Rhodococcus rhodochrous ATCC 21198] & $\begin{array}{l}\text { glyoxylate/dicarboxylate } \\
\text { metabolism }\end{array}$ & gi|573523598 & $47 \mathrm{kDa}$ \\
\hline 12.0 & 0.0013 & catechol 1,2-dioxygenase [Rhodococcus rhodochrous ATCC 21198] & Phenol degradation & gi|573524225 & $32 \mathrm{kDa}$ \\
\hline 16.0 & $<0.00010$ & Transaldolase [Rhodococcus rhodochrous ATCC 21198] & central metabolism & gi|573525361 & $41 \mathrm{kDa}$ \\
\hline 16.0 & $<0.00010$ & 3-hydroxybutyryl-CoA epimerase [Rhodococcus rhodochrous ATCC 21198] & fatty acid metabolism & gi|573526670 & $75 \mathrm{kDa}$ \\
\hline 22.0 & $<0.00010$ & putative $\mathrm{HpaB} / \mathrm{PvcC} / 4-\mathrm{BUDH}$, partial [Rhodococcus rhodochrous ATCC 21198] & Phenol degradation & gi|573528977 & $36 \mathrm{kDa}$ \\
\hline
\end{tabular}

